\title{
Economics of Leadership and Hierarchy
}

\author{
Junjie Zhou *
}

August 2012

\begin{abstract}
This paper explores leadership within hierarchical organizations. For each hierarchy, I consider a dynamic signaling game in which each player observes only the actions of his direct superiors before choosing his action. At the top of the hierarchy are the leaders, who learn the state from nature. The hierarchy controls the flow of information and the timing of the game, and determines the equilibrium output and welfare. I show that the welfareoptimal hierarchy is the chain, because it maximizes the incentive of players to "lead by example" for their subordinates. The chain remains optimal even in the presence of verifiable or unverifiable costly information acquisition by the leaders. Lastly, I characterize optimal hierarchies when the number of layers or the number of leaders is limited. Applications to fund-raising are also discussed.
\end{abstract}

Keywords: organization design, hierarchy, leadership, free-riding, dynamic signaling, lead by example

JEL Numbers: D21, D29, D82, L29

* School of International Business Administration, Shanghai University of Finance and Economics, 777 Guoding Road, Shanghai, China 200433. E-mail: zhoujj03001@gmail.com. I am indebted to Professors Benjamin Hermalin, Chris Shannon and Suzanne Scotchmer for their continuous guidance and advice during the course of this project. I also thank Professors Ying-Ju Chen, Joan de Martí Beltran, Thomas Marschak, Alessandro Pavan and Adam Szeidl and the seminar participants at UC Berkeley, 2012 North American Summer Meeting of the Econometric Society, 2012 Shanghai Microeconomics Workshop for helpful comments. All errors are mine. 


\section{Introduction}

This paper studies the role of leadership and information flow in the design of organizations. I develop a model of public good provision in teams with asymmetric information. Team members can engage in costly signaling of their information through their choice of effort to invest in the joint project. Leadership positions within the organization are distinguished by differential access to information: a team member's effort is observed only by her direct subordinates. The flow of information is thus endogenous to the design of the organization, and becomes the crucial channel through with the organizational design affects team output. I characterize the optimal organizational design in this model, and show that the optimal hierarchy provides important welfare gains over the standard team output and other methods of addressing the classic problem of moral hazard in teams (Holmstrom (1982)).

A central building block for my work is the idea of leading by example, introduced in the seminal work of Hermalin (1998). Hermalin (1998) also starts from the issue of free-riding in team production problems, and assumes that one team member knows the true marginal return to effort. In the standard team model, the informed member cannot credibly signal her information, thus it is useless. Hermalin's fundamental insight is that if the informed member can move first, however, then she can "lead by example": if she chooses her effort first and this is observable to all other team members, then her investment in the project provides a credible costly signal. Hermalin (1998) shows that such leading by example, by exploiting this information channel, yields higher welfare in equilibrium than the standard team production (even in the symmetric information case). Thus Hermalin (1998) identifies an important aspect of leadership in information transmission and incentive provision that can mitigate free-riding in teams. This insight has been at the heart of a sizable and growing literature on the economics of leadership (for example, see Hermalin (2007), Komai, Stegeman and Hermalin (2007), Komai and Stegeman (2010)).

An important limitation to the analysis in Hermalin (1998) is that the information channels and organizational structure are essentially taken to be exogenous. One team member is exogenously assumed to be informed about the true state, 
thus have "leadership potential." The leader's only choice is whether to move first, thereby signaling to all of the other members simultaneously. Thus the organizational structure is exogenously given: a two-tier hierarchy with the leader at the top and all other members on the second tier. If the signaling role of a leader is important, however, then information flows should be an important component in the endogenous design of organizations. For example, consider a three-person team in which one member learns the true state. Hermalin's (1998) results show that team output increases if the informed member invests first and reveals her investment to the other members, who then choose their investments simultaneously. Is this the optimal organizational design, however? Hermalin (1998) and the substantial work that followed do not address this important question. For example, is it better to have information flow through a "middle manager," that is, to have a three-tier hierarchy in which a single member observes the leader's investment, chooses his investment and then in turn reveals only his investment to the third member? Or is it better to have two leaders, each of whom signals to the third member?

To answer these questions, I start by illustrating the results in the simple case of three workers. In this case, it is possible to give an exhaustive list of all of the possible hierarchies. In a simplified version of the public good provision model with quadratic disutility of effort, I show that the optimal hierarchy has three tiers, with one leader on the top tier, one middle manager on the second tier, and a terminal worker on the third tier. This results in two rounds of the "leading by example" effect observed by Hermalin (1998). The middle manager "leads by example" for the terminal worker, which results in higher effort from the middle manager due to the need to provide credible signal. Because the middle manager works harder, the leader has a larger incentive to invest more as well, again due to the signaling effect. In particular, this three-tier chain hierarchy yields higher output than the two-tier hierarchy assumed in Hermalin (1998). Similarly, the chain dominates the inverted two-tier hierarchy with two leaders on the top tier ${ }^{1}$ In either case, one round of signaling is wasted, leading to lower output than in the chain.

\footnotetext{
${ }^{1}$ This result relies on an assumption on the beliefs of the terminal worker. See Section 2.3.
} 
The general model with any number of workers and general sharing rule and disutility function is analyzed in sections 4 and 5 . Here I distinguish between simple hierarchies, in which every player who is not the leader has a unique direct predecessor, and complicated hierarchies, in which at least one player has multiple direct predecessors. In a simple hierarchy, the dynamic signaling game I define always has a unique separating equilibrium, but in a complicated hierarchy typically there are multiple separating equilibria. Consequently, the analysis of complicated hierarchies is more delicate.

For simple hierarchies, I show that similar intuition as in the three-person example holds, and the chain is optimal in an arbitrarily large team. The optimality of the chain follows from the observation that by transforming any hierarchy into a chain, I obtain the maximal number of stages of signaling, as the set of followers for each member is larger in the chain than in the original hierarchy. For fixed shares, the chain gives every member the largest possible signaling incentive, hence motivates the highest efforts. Therefore, the chain can replicate the same welfare under any hierarchy but uses less total shares. Moreover, extra shares always improve welfare when distributed optimally among the team. Combining these results shows the optimality of the chain among simple hierarchies.

For complicated hierarchies, I focus on a particular equilibrium which shares a similar characterization as the unique equilibrium with simple hierarchies. Here I consider two operations on hierarchies: adding links and splitting. Adding links means constructing a link, and hence an information channel, between two members who were unconnected, while splitting means creating a new intermediate tier consisting of a single member chosen from a tier with more than one member, and adding the maximal number of links to this new tier. Interestingly, each operation improves welfare after adjusting the shares optimally. The optimality of the chain follows directly from the fact that any hierarchy can be transformed into a chain through a sequence of these two operations.

I then extend the model to allow for endogenous information acquisition by the leaders. If research effort is verifiable, then the optimal hierarchy is still the chain because the chain generates the highest social return to information. Even if research effort is not verifiable, the chain remains optimal because the leader's incentive for information acquisition now depends monotonically on her equilibrium 
effort, which is higher in the chain than in any other hierarchy. Thus the leader acquires more accurate information in equilibrium, even when research effort is not verifiable.

A drawback to the chain is that for a large team, the hierarchy is very long, as it requires as many tiers as team members. Thus I also consider a version of the model with constraints on the height of the hierarchy, that is, in which hierarchies are constrained to have fewer tiers than team members. In this case a chain is not feasible. I show that the optimal simple hierarchy must have the maximal number of middle managers, hence the smallest number of terminal workers. This is achieved by assigning at most one follower to each middle manager. The maximal number of middle managers exploits the maximal level of signaling incentives in the team when height is limited.

While I use the language of leaders and followers throughout the paper, following Hermalin (1998) and subsequent work, the results developed here can be applied to a wide variety of team production problems with asymmetric information. In many applications, the informed players who move first in the optimal team hierarchy need not literally be team "leaders" or CEOs; in many settings it might be natural for more informed members to be lower-level workers more familiar with the production technology or better able to collect information. In such problems, these results show that the optimal arrangement of the team is a chain originating with the informed member, with each member signaling via his effort to a subsequent member.

As an application, I consider the problem of a charity trying to raise funds from a pool of possible donors. I show that the charity can raise more money by implementing a fund-raising campaign resembling a chain; that is, by placing potential donors in a line and asking them to donate one after the other. In particular, the charity should not reveal the entire donation history to future donors.

\section{Related Literature}

This paper is related to two strands of literature, one focusing on the economics of leadership, and the other focusing on determinants of organizational design. 
As mentioned an important contribution to the literature of leadership is Hermalin (1998), on which this paper is built. Many extensions of Hermalin's model have appeared. Komai and Stegeman (2010) study the rise of leaders endogenously. Komai, Stegeman, and Hermalin (2007) consider team production with binary action (participate or not). Hermalin (2008) extends the static model to an infinitely repeated setting, thus allowing the leader to build a reputation. The literature on leaders conveying information is surveyed in Hermalin (2007). As noted, a limitation of these models is that they all assume an exogenous organizational structure. The main contribution of this paper is to endogenize the organizational structure. In a recent paper, Acemoglu and Jackson (2011) study how the prominent agents, the leaders whose actions are perfectly observed by the followers, can drive the changes in social norms in a dynamic game. However, the chain sequence of moves for agents is exogenously assumed.

Many different aspects of hierarchies have been studied in previous work. Some approaches emphasize moral hazard and loss of control, for example, Calvo and Wellisz (1978, 1979), and Qian (1994). Others, following Radner (1993), study optimal hierarchies for minimizing costs of information processing and communication, for example, Bolton and Dewatripont (1994), Prat (1997), van Zandt (1999), Marschak and Reichelstein (1998). This paper identifies another role of hierarchy through a different perspective, that of signaling channels. The dissemination of information along the hierarchy creates incentives for players as they try to influence their followers' beliefs, hence efforts.

Closer to the analysis of my model is Winter (2010)2 who studies the effect of transparency among peers on the principal's cost of providing incentives in a moral hazard model. He uses direct graph to model transparency among peers, and shows that it's cheaper to provide incentives under more transparency, thus proves the optimality of the chain structure. Though these two paper look similar at first glance, there are three main differences: First, the production function in Winter's paper exhibits a high degree of complementarity, which drives the main results in his paper, while the production function in my paper is additively separable, thus there is no explicit complementarity or synergy among

\footnotetext{
${ }^{2}$ See also Winter (2004), Winter (2006).
} 
workers. Second, in Winter (2010), the first-mover's action affects his follower's efforts through complementarity in the production function, while in my paper the effort of the first-mover is acting as a signal about the state, the profitability of the project, to the latter-movers, and influences the beliefs, thus efforts of the followers through a totally different mechanism. Third, the objectives of these two papers are different. Winter compares the cost of implementing a certain action profile in a team under various internal information about effort, while I characterize the welfare-maximizing hierarchical structure in an organization.

This paper is also related to the increasingly growing literature on social learning (Bala and Goyal, 1998) and social networks (Jackson, 2008). The hierarchies studied here are particular networks in which information is transmitted through signaling along the directed links. In this model, cheap talk messages are not credible. Instead, the information about the state is transmitted from one player to his follower via his action, which connects this paper to the literature on "information cascades" and "herd behavior" (see, e.g., Banerjee 1992, Bikhchandani et al., 1992). But there are many differences. First, followers in this paper have no private signals. Also, the action and state are both continua, the action fully reveals the state in equilibrium. Lastly, unlike that literature, one player's payoff here also depends on other players' actions.

\section{Model with Three Workers}

In this section, I provide basic intuition about how organizational structure affects the incentives of players and team welfare using a simplified three-worker public good production model. The general model with any fixed number of workers, general sharing rule and disutility function is studied in the next section.

For an organization with three workers, there are only a few possible hierarchies: T structure (team structure), $\Lambda$ structure (leading by example), $C_{3}$ (sequential leading by example), and V structure (two leaders) $\mathrm{H}^{3}$ For each hierarchy, I define the game associated with it, characterize the unique separating equilibrium, and compute welfare in that equilibrium. I show the chain yields higher

\footnotetext{
${ }^{3}$ There is another structure with two leaders, but one leader has no followers. See footnote 7 for discussion.
} 
welfare than leading by example, which in turn yields higher welfare than the team structure. The analysis for the $\mathrm{V}$ structure is complicated by the fact that the unique follower of two leaders may have different out-of-equilibrium beliefs to support different equilibrium efforts from the leaders. Under a pessimistic belief assumption for the follower, I show that there is a continuum of separating equilibria, but all such equilibria are bounded by two special equilibria, what I call the U-equilibrium and L-equilibrium. The U-equilibrium does better than leading by example, but still worse than the chain, while the L-equilibrium does as well as the team structure. Hence, I give a complete picture of what can achieve with three workers.

Consider a team with $\mathrm{N}=3$ identical workers producing a joint project. The value of the project is $v\left(x_{1}, x_{2}, x_{3}\right)=\theta\left(x_{1}+x_{2}+x_{3}\right)$, where $\theta \in \Theta=[0, \infty)$ is a stochastic productivity factor and $x_{i} \in \mathbf{R}_{+}$is the contribution of worker $i$. The prior distribution of $\theta$ is $F:[0, \infty) \rightarrow[0,1]$. I assume $F$ has full support and a continuous and positive density function $f$. Furthermore, assume each member gets $1 / 3$ of the total output $v$ and $c(x)=\frac{1}{2} x^{2}$ is the disutility of effort, which is the same for every worker. Then, worker $i$ 's payoff $\pi_{i}$ is $\pi_{i}\left(x_{1}, x_{2}, x_{3}\right)=\frac{1}{3} v\left(x_{1}, x_{2}, x_{3}\right)-c\left(x_{i}\right)=\frac{1}{3} \theta\left(x_{1}+x_{2}+x_{3}\right)-\frac{1}{2} x_{i}^{2}$ and aggregate welfare is $W\left(x_{1}, x_{2}, x_{3}\right)=\sum_{i=1}^{3} \pi_{i}\left(x_{1}, x_{2}, x_{3}\right)=\sum_{i=1}^{3}\left(\theta x_{i}-\frac{1}{2} x_{i}^{2}\right)$. Note that the output function $v$ is additively separable in individual efforts. So, if state $\theta$ is common knowledge or all workers have the same belief $\theta$ about the state, worker $i$ has a dominant strategy to exert effort $x_{i}^{N}=\frac{1}{3} \theta$. Welfare under the corresponding equilibrium is $W^{N}(\theta)=W\left(\frac{1}{3} \theta, \frac{1}{3} \theta, \frac{1}{3} \theta\right)=\frac{5}{6} \theta^{2}$. The first-best effort is $x_{i}^{F B}=\theta$ and the first-best welfare is $W^{F B}(\theta)=W(\theta, \theta, \theta)=\frac{3}{2} \theta^{2}$. There is under-provision of effort due to standard free-riding in teams. In the first-best world, each worker must receive $100 \%$ of the output on the margin, but in total I only have $100 \%$ to give due to budget balance. The case with symmetric information can be graphically represented by Figure 1. Every member's position is symmetric in this structure, and I call it the standard team structure.

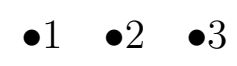

Figure 1: Standard Team Structure (T) 


\section{$2.1 \quad$ Leading by Example}

To counteract the free-riding problem in teams, hidden information and leading by example were introduced by Hermalin (1998). In that model, one worker, called the "leader," has superior information about $\theta$ and she moves first. All of the other workers, who initially only know the prior distribution of the state, observe her effort and choose their efforts simultaneously in the next stage.

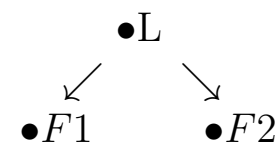

Figure 2: Leading by Example $(\Lambda)$

Figure 2 shows the relationship between workers. L is the leader and $F 1$ and $F 2$ are the two followers. An arrow from L to F1 means that F1 observes L's effort. I call this the $\Lambda$ structure since it resembles the letter $\Lambda$. For the $\Lambda$ structure, I can define a signaling game as follows:

- Nature chooses $\theta \in \Theta$, which is unknown to all initially.

- In period 1 , the leader L learns $\theta$ and chooses $x_{L}$.

- In period 2, both F1 and F2 observe $x_{L}$ and pick their efforts $x_{F 1}, x_{F 2}$ simultaneously.

- Payoffs are realized.

I am interested in separating equilibrium, in which the leader's effort $\tilde{x}_{L}(\theta)$ is a monotonic function of the state $\theta$, hence reveals the state to the followers. A separating Perfect Bayesian Equilibrium (SPBE) of this signaling game is calculated in Hermalin (1998, Lemma 4). In that equilibrium, the leader's equilibrium strategy is $\tilde{x}_{L}(\theta)=\frac{2}{3} \theta$. After observing the leader's effort $x_{L}$, each follower's point belief about the state is $\beta_{i}\left(x_{L}\right)=\frac{3}{2} x_{L}, i=1,2$. Hence, each follower chooses effort $x_{F_{i}}\left(x_{L}\right)=\frac{1}{3} \beta_{i}\left(x_{L}\right)=\frac{1}{2} x_{L}, i=1,2$. The equilibrium efforts of $L, F 1, F 2$ are $\frac{2}{3} \theta, \frac{1}{3} \theta$, and $\frac{1}{3} \theta$ respectively. 
The equilibrium welfare in this equilibrium is $W^{L}(\theta)=W\left(\frac{2}{3} \theta, \frac{1}{3} \theta, \frac{1}{3} \theta\right)=\theta^{2}$ So, the welfare with leading by example is higher than that with symmetric information, i.e, $W^{L}=\theta^{2}>W^{N}=\frac{5}{6} \theta^{2}$ Compared with the team structure, leading by example improves welfare, because the equilibrium effort of the leader is larger than under symmetric information, but still below the first-best level. The intuition is as follows. The leader gets a portion of the output generated by followers. In a separating equilibrium, the leader's effort fully reveals the information about the state. The harder the leader works, the higher the followers' beliefs about the state, thus the harder followers work and the better off is the leader. Being a leader and signaling to the followers gives leader L extra incentive to work hard beyond the incentive from her own share of the output. Given that the equilibrium efforts with symmetric information are too low to begin with, inducing harder work is welfare improving.

\subsection{Sequential leading by example}

Leading by example improves welfare in teams as shown by Hermalin (1998). But in equilibrium, efforts of the followers F1 and F2 are still too low. Welfare would be even higher if I could motivate any of the followers to exert higher effort in equilibrium. This requires that the followers have some extra signaling incentive as the leader $\mathrm{L}$ had in the previous example. This leads us to change the $\Lambda$ structure into a chain (see Figure 3). The timing of the game is now modified as follows. In period 2, only F1 (not F2 ) can observe L's effort $x_{L}$, and he exerts effort in period 2. In period 3, F2 observes F1's effort $x_{F 1}$ and exerts effort $x_{F 2}$ last.

$$
\bullet \mathrm{L} \rightarrow \bullet F 1 \rightarrow \bullet F 2
$$

Figure 3: The Chain Structure $\left(C_{3}\right)$

The chain structure contains two stages of leading by example. Leader L signals to F1, and F1 signals to F2. Critically, F2 cannot observe the leader L's effort

\footnotetext{
${ }^{4}$ This is true except when $\theta=0$. For the sake of brevity, I will not repeat this caveat later.
} 
directly in period 2, otherwise F1 would have no signaling incentive because F2 would have already known the state from L's effort.

A separating Perfect Bayesian Equilibrium (SPBE) of this game is a strategy profile $\left\langle\tilde{x}_{L}(\cdot), \tilde{x}_{F 1}(\cdot), \tilde{x}_{F 2}(\cdot)\right\rangle$ and beliefs $\beta_{F 1}(\cdot), \beta_{F 1}(\cdot)$ such that:

(S) All $\tilde{x}_{i}, i \in\{L, F 1, F 2\}, \beta_{j}, j \in\{F 1, F 2\}$ are monotonic.

$(P L) \quad \forall \theta, \tilde{x}_{L}(\theta) \in \arg \max _{x_{L} \geq 0} \frac{\theta}{3}\left(x_{L}+\tilde{x}_{F 1}\left(x_{L}\right)+\tilde{x}_{F 2}\left(\tilde{x}_{F 1}\left(x_{L}\right)\right)\right)-\frac{1}{2} x_{L}^{2}$.

$(P 1) \quad \forall x_{L}, \tilde{x}_{F 1}\left(x_{L}\right) \in \arg \max _{x_{F 1} \geq 0} \frac{\beta_{F 1}\left(x_{L}\right)}{3}\left(x_{L}+x_{F 1}+\tilde{x}_{F 2}\left(x_{F 1}\right)\right)-\frac{1}{2} x_{F 1}^{2}$.

$(P 2) \quad \forall x_{F 1}, \tilde{x}_{F 2}\left(x_{F 1}\right) \in \arg \max _{x_{F 2} \geq 0} \frac{\beta_{F 2}\left(x_{F 1}\right)}{3}\left(x_{L}+x_{F 1}+x_{F 2}\right)-\frac{1}{2} x_{F 2}^{2}$.

and

(B) $\quad \beta_{F 1}=\tilde{x}_{L}^{-1}$, and $\beta_{F 2}=\beta_{F 1} \circ \tilde{x}_{F 1}^{-1}$

$\mathrm{S}$ says that each player's effort fully reveals his belief about the state under any history. B specifies the belief updating rule. $P L, P 1$, and $P 2$ are the usual perfection conditions: Each player is acting optimally given other players' beliefs and best responses.

It is easy to check that the following is a SPBE of this game:

$$
\begin{gathered}
\tilde{x}_{L}(\theta)=k_{L} \theta, \\
\beta_{F 1}\left(x_{L}\right)=\frac{x_{L}}{k_{L}} \quad \tilde{x}_{F 1}\left(x_{L}\right)=k_{F 1} \beta_{F 1}\left(x_{L}\right)=\frac{k_{F 1}}{k_{L}} x_{L} \\
\beta_{F 2}\left(x_{F 1}\right)=\frac{x_{F 1}}{k_{F 1}} \quad \tilde{x}_{F 2}\left(x_{F 1}\right)=k_{F 2} \beta_{F 2}\left(x_{F 1}\right)=\frac{k_{F 2}}{k_{F 1}} x_{F 1}
\end{gathered}
$$

The constants $k_{i}, i=\{L, F 1, F 2\}$ are given by

$$
k_{F 2}=\frac{1}{3} \approx 0.333, \quad k_{F 1}=\frac{1+\sqrt{5}}{6} \approx 0.539, \quad k_{L}=\frac{1+\sqrt{7+2 \sqrt{5}}}{6} \approx 0.731
$$

The equilibrium effort of player $i$ is $k_{i} \theta, i=\{L, F 1, F 2\}$ and the corresponding welfare is

$$
W^{S}=W\left(k_{L} \theta, k_{F 1} \theta, k_{F 2} \theta\right) \approx 1.13554 \theta^{2}
$$

\footnotetext{
${ }^{5}$ This result is a special case of Theorem 4.1 .
} 
It is clear that $W^{S}>W^{N}$, so I have established that the chain structure (Figure 3 with sequential leadership yields greater welfare than the $\Lambda$ structure (Figure 2) with a single leader.

This result can be interpreted in the following way. In the chain structure, F1 plays leading by example with F2 as F2 infers the state from his effort. Due to signaling, F1's response as a function of his belief about the state must be steeper than what he chooses when he has no followers. Note that the leader $\mathrm{L}$ cannot influence the beliefs of player $F 2$ directly as $F 2$ cannot observe his effort, but indirectly L can influence the belief and effort of F2 through the intermediate player F1.

The leader L benefits from output generated by both F1 and F2. Her effort provides the information about the state. The greater her effort, the higher F1's belief, the greater F1's effort, the higher F2's belief, the higher F2's effort, and the better off is the leader. But the difference is now that F1's response given his belief $\beta_{1}$ is steeper under the chain than under the $\Lambda$ structure. Therefore, to determine L's incentive for signaling, we can imagine that there is just one stage of signaling, as in Figure 2, with the share of F1 modified to $k_{F 1} \approx 0.539>1 / 3$. This extra benefit increases the leader's equilibrium effort, thus we obtain $k_{L}>2 / 3$. Since all the equilibrium efforts are still below first-best levels, I have obtained our welfare-comparison result.

At this time, it is worthwhile to check the role of additively separable production functions in this model. First, additive separability isolates the signaling effects from other forces possibly driven by strategic complements or substitutes between workers. If information is symmetric, the timing of moves is irrelevant as everyone has a dominant strategy, thus organizational structure does not play any role. When information is asymmetric, all the welfare comparison results must come from the signaling incentives. Second, additive separability greatly simplifies the computation of equilibrium. The strategic role of effort in this model is that it conveys valuable information about the state from one player to his followers. In the dynamic signaling game defined above, each worker's incentive consists of two parts. The first (direct) part is his share of the output. The second (indirect) part is strengthening of incentives of workers whose beliefs he can influence by his own effort, either directly or indirectly. In the chain, F1's effort can only affect 
F2's belief, while L can influence F1's belief directly and F2's belief indirectly. F2 cannot influence anyone's belief and therefore the second incentive component is zero. The higher is each incentive part, the harder one works.

\subsection{Two leaders}

Beyond the chain and the $\Lambda$ structure, there is another hierarchy, called the $\mathrm{V}$ structure, possible with three workers. In the V structure, L1 and L2 are leaders and $\mathrm{F}$ is the only follower of both. See Figure 4. The time line for the V structure

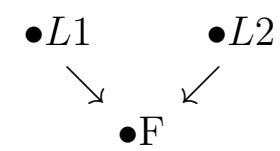

Figure 4: V structure, with two leaders

is the following:

- Nature chooses $\theta \in[0, \infty)$, which is unknown to all.

- In period 1 , both leaders L1 and L2 learn $\theta$ and choose $x_{1}, x_{2}$ simultaneously.

- In period 2, F observes both leaders' efforts $x_{1}, x_{2}$ and exerts effort $x_{F}$.

- Payoffs are realized.

A SPBE of this game is a strategy profile $\left\langle e_{1}(\cdot), e_{2}(\cdot), e_{F}(\cdot, \cdot)\right\rangle$ and belief function $b_{F}(\cdot, \cdot)$ such that:

(S) $e_{i}(\theta), i=\{1,2\}$ are monotonic.

$(P 1) \quad \forall \theta, e_{1}(\theta) \in \arg \max _{x_{1} \geq 0} \frac{\theta}{3}\left(x_{1}+e_{F}\left(x_{1}, e_{2}(\theta)\right)+e_{2}(\theta)\right)-\frac{1}{2} x_{1}^{2}$.

$\left.(P 2) \quad \forall \theta, e_{2}(\theta) \in \arg \max _{x_{2} \geq 0} \frac{\theta}{3}\left(e_{1}(\theta)+e_{F}\left(e_{1}(\theta), x_{2}\right)+x_{2}\right)\right)-\frac{1}{2} x_{2}^{2}$.

$(P F) \quad \forall x_{1}, x_{2} \geq 0, e_{F}\left(x_{1}, x_{2}\right) \in \arg \max _{x_{f} \geq 0} \frac{b_{F}\left(x_{1}, x_{2}\right)}{3}\left(x_{1}+x_{2}+x_{f}\right)-\frac{1}{2} x_{f}^{2}$.

(B) $\forall \theta, b_{F}\left(e_{1}(\theta), e_{2}(\theta)\right)=\theta$. 
$\mathrm{P} 1$ and $\mathrm{P} 2$ and $\mathrm{PF}$ are the perfection conditions. Note that B says that the belief of $\mathrm{F}$ is correct on the equilibrium path, but it is silent about F's out-ofequilibrium beliefs.

Obviously, PF could be replaced by

$$
\left(P F^{\prime}\right) \quad e_{F}\left(x_{1}, x_{2}\right)=b_{F}\left(x_{1}, x_{2}\right) / 3
$$

For convenience, define $\beta: \Theta \times \Theta \rightarrow \Theta$ by $\beta\left(\theta_{1}, \theta_{2}\right):=b_{F}\left(e_{1}\left(\theta_{1}\right), e_{2}\left(\theta_{2}\right)\right)$. Then $b_{F}\left(x_{1}, x_{2}\right)=\beta\left(e_{1}^{-1}\left(x_{1}\right), e_{2}^{-1}\left(x_{2}\right)\right)$. After simplifying and using $\left(P F^{\prime}\right)$, we can rewrite conditions $\mathrm{P} 1$ and $\mathrm{P} 2$ and $\mathrm{B}$ as:

$$
\begin{aligned}
& \left(P 1^{\prime}\right) \quad \forall \theta, e_{1}(\theta) \in \arg \max _{x_{1} \geq 0} \frac{\theta}{3}\left(x_{1}+\frac{1}{3} \beta\left(e_{1}^{-1}\left(x_{1}\right), \theta\right)\right)-\frac{1}{2} x_{1}^{2} . \\
& \left(P 2^{\prime}\right) \quad \forall \theta, e_{2}(\theta) \in \arg \max _{x_{2} \geq 0} \frac{\theta}{3}\left(x_{2}+\frac{1}{3} \beta\left(\theta, e_{2}^{-1}\left(x_{2}\right)\right)\right)-\frac{1}{2} x_{2}^{2} . \\
& \left(B^{\prime}\right) \quad \forall \theta, \beta(\theta, \theta)=\theta .
\end{aligned}
$$

To find an equilibrium, it suffices to find functions $\left\{e_{1}, e_{2}, \beta\right\}$ satisfying conditions $S, P 1^{\prime}, P 2^{\prime}, B^{\prime}$. There are many belief functions satisfying $B^{\prime}$. I will use the following pessimistic belief assumption.

$$
\beta^{p}\left(\theta_{1}, \theta_{2}\right)=\min \left(\theta_{1}, \theta_{2}\right)
$$

There are two main justifications for this assumption. First, it gives both leaders some incentive to signal to the follower. If any of the leaders shirks, the follower will detect it immediately and punish that deviating leader by expending lower effort because the follower believes that the minimum of the two signals, here the one revealed by the deviating leader, is the true state. Second, if the follower's belief is the maximum of the two signals, then it is impossible to support any separating equilibrium, because at least one leader will have incentive to deviate either downward (free-riding on the other leader to signal to the follower) or upward (taking advantage of the benefits of signaling as the follower, in this case, will depend solely on his effort for updating beliefs) ${ }^{6}$ If the follower believes that off the equilibrium path only one of the leaders is deviating, which is mostly likely to be the case, the pessimistic belief function is a better choice as the optimistic

\footnotetext{
${ }^{6}$ See appendix section C.2 for a detailed proof.
} 
belief cannot support any equilibrium. Hence, we will maintain this pessimistic belief assumption from now on. For detailed discussions of the belief functions in the $V$ structure, see the appendix.

$\operatorname{Fix} \beta=\beta^{p}$. Let $\bar{e}(\theta):=\frac{1+\sqrt{5}}{6} \theta$ and $\underline{e}(\theta):=\frac{\theta}{3}$. It is easy to verify that $e_{i}=\bar{e}(\theta)$, $i=1,2$, or $e_{i}=\underline{e}(\theta), i=1,2$ satisfy the above conditions $\left(P 1^{\prime}\right),\left(P 2^{\prime}\right),\left(B^{\prime}\right)$ with $\beta=\beta^{p}$. I call the equilibrium with $e_{i}=\bar{e}(\theta)(\underline{e}(\theta)), \forall i$ the $\mathrm{U}(\mathrm{L})$-equilibrium. In the U-equilibrium, both leaders exert higher efforts $\bar{e}$, while in the L-equilibrium, both leaders choose $\underline{e}=\frac{1}{3} \theta=x_{i}^{N}$, their efforts under symmetric information. In the appendix, I show that all separating equilibria corresponding to $\beta^{p}$ are bounded by these two equilibria. Therefore, I can find the upper and lower bounds of the corresponding equilibrium welfare:

$$
\frac{5}{6} \theta^{2} \leq W^{2 L} \leq \frac{(8+5 \sqrt{5})}{18} \theta^{2}
$$

The lower bound corresponds to the L-equilibrium, while the upper bound corresponds to the U-equilibrium. Any number in between is also obtainable.

\subsection{Welfare comparison}

In previous subsections, I solve for equilibrium under four different structures with three workers. The computed welfares are ranked as follows:

$$
W^{N}=\min W^{2 L}<W^{L}<\max W^{2 L}<W^{S}<W^{F B}
$$

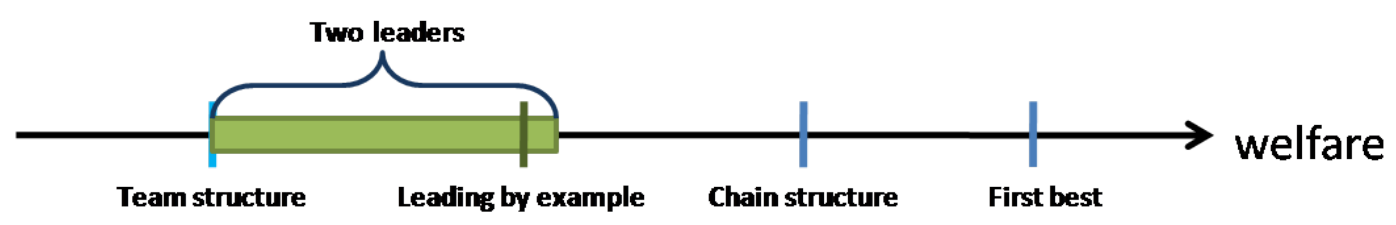

Figure 5: Welfare Rankings

As a conclusion, the chain structure dominates each of the other three structures $]^{7}$ None of them can achieve first-best welfare, however. Figure 5 summarizes these results.

\footnotetext{
${ }^{7}$ Technically, there is one more structure with three workers constructed by deleting the link from $\mathrm{L} 2$ to $\mathrm{F}$ in the $\mathrm{V}$ structure. It is easy to see that this structure is less efficient than the $\Lambda$ structure, hence worse than the chain.
} 
As mentioned, workers on the higher tier of a chain exert higher efforts. One concern is that their efforts are inefficiently high, possibly in a team with large $N$. Part of the following analysis is to demonstrate that this phenomena is not going to happen in a team with arbitrary $N$. In the next section, I study the general model, and prove the optimality of the chain in the end.

\section{The General Model}

Let $\mathcal{N}=\{1,2, \cdots, N\}$. Consider a team with $N$ identical members. Each member $n$ chooses an effort $e_{n} \in[0, \infty)$. The value to the team is $V=\theta \sum_{n=1}^{N} e_{n}$, where $\theta \in \Theta=[0, \infty)$ is a stochastic productivity factor. Each member has utility function $w-c(e)$, where $w$ is his wage, and the disutility function $c$ is twice differentiable and strictly convex with $c(0)=c^{\prime}(0)=0, c^{\prime}(\infty)=\infty$.

Following Holmstrom (1982), I assume that contracts can only be written contingent on total output $V$, not on individual efforts. Furthermore, I restrict attention to affine-shares contracts, i.e., $w_{n}(V)=s_{n} V+t_{n}$. Here $\left\{s_{n}, t_{n}\right\}_{n=1}^{N}$ are constants. No external source of funds means that $\sum_{n=1}^{N} w_{n} \leq V$. Requiring contracts to be renegotiation-proof means $\sum_{n=1}^{N} w_{n} \nless V$. Therefore $\sum_{n=1}^{N} s_{n}=1, s_{n} \geq 0$, and $\sum_{n=1}^{N} t_{n}=0$. I am interested in team members' equilibrium efforts. The transfers $\left\{t_{n}\right\}$ are irrelevant to our analysis, hence omitted in our calculations. Let $\Delta^{N}=\left\{\mathbf{s}=\left(s_{1}, \cdots, s_{N}\right) \mid \sum_{i=1}^{N} s_{i}=1, s_{i} \geq 0, \forall i\right\}$ denote the $N$-dimensional simplex. 8 Then an affine-shares contract is just an element $\mathbf{s}$ of $\Delta^{N}$.

As we have seen in section 2, hierarchy matters for the performance of the team. To define a hierarchy formally, I first review some concepts from graph theory.

Definition 1 (Graph) A directed graph $(\mathcal{N}, \mathbf{d})$ consists of a set of nodes $\mathcal{N}=$ $\{1,2, \cdots, N\}$ and an adjacency matrix $\mathbf{d}=\left(d_{i j}\right)_{N \times N} . d_{i j}=1$ if there is a directed link from $i$ to $j$, otherwise $d_{i j}=0$.

$A$ path from $i$ to $j$ is a sequence of nodes $i_{1}, i_{2}, \cdots, i_{k}$ such that $i_{k}=j$, and $d_{i i_{1}}=d_{i_{2} i_{3}}=\cdots d_{i_{k-1} i_{k}}=1$, while $k$ is called the length of this path.

\footnotetext{
${ }^{8}$ Later on, I will allow $\sum_{n=1}^{N} s_{i} \leq 1$ in some of the proofs.
} 
In this paper, $\mathcal{N}$ is fixed, thus I refer to $\mathbf{d}$ as a graph. For a fixed adjacency matrix d, I can define $D F^{i}=\left\{j \in \mathcal{N} \mid d_{i j}=1\right\}$ as the set of direct followers of $i$, and $F^{i}=\{j \in \mathcal{N} \mid$ there is a path from $i$ to $j\}$ as the set of $i$ 's followers, direct and indirect. Similarly, I define $D P^{i}=\left\{j \in \mathcal{N} \mid d_{j i}=1\right\}$ as the set of direct predecessors of $i$, and $P^{i}=\{j \in \mathcal{N} \mid$ there is a path from $j$ to $i\}$ as the set of $i$ 's predecessors, direct and indirect. By definition, $j \in D F^{i}$ if and only if $i \in D P^{j}$. Similarly, $j \in F^{i}$ if and only if $i \in P^{j}$. Obviously $D F^{i} \subset F^{i}$ and $D P^{i} \subset P^{i}, \forall i$.

Definition 2 (Ordered Partition) $\mathcal{P}=\left\{A^{1}, A^{2}, \cdots, A^{m}\right\}$ is called an ordered partition of $A$ if (1) $A^{i} \neq \emptyset$ for all $i$; (2) $\cup_{i=1}^{m} A^{i}=A$; (3) $A^{i} \cap A^{j}=\emptyset, \forall i \neq j$.

Definition 3 (Hierarchy) $A$ hierarchy $\mathcal{H}=(\mathbf{d}, \mathcal{P})$ on $\mathcal{N}$ is a directed graph $(\mathcal{N}, \mathbf{d})$ together with an ordered partition $\mathcal{P}=\left\{N^{1}, N^{2}, \cdots, N^{h}\right\}$ of $\mathcal{N}$ such that:

(a) for $k=1,2, \cdots, h-1$, if $j \in N^{k}$ and $d_{j i}=1$, then $i \in N^{k+1}$. If $j \in N^{h}$, then $d_{j i}=0, \forall i$.

(b) for $k=2, \cdots, h$, if $j \in N^{k}$, then there is a node $i \in N^{k-1}$ such that $d_{i j}=1$.

Definition 4 In a hierarchy $\mathcal{H}=(\mathbf{d}, \mathcal{P})$ with $\mathcal{P}=\left\{N^{1}, N^{2}, \cdots, N^{h}\right\}$, the number $h$ is called the height of $\mathcal{H}$.

For a fixed hierarchy $\mathcal{H}, N^{k}$ is just the set of members on level $k$, and $n^{k}=\left|N^{k}\right|$ is the number of workers on level $k$. The workers in $N^{1}$ are called the leaders, while the workers in $N^{h}$ are called terminal workers.

Condition (a) means that a path can only connect nodes from one level to the next lower level, while condition (b) says that all workers except leaders have at least one predecessor. Terminal workers have no followers and leaders have no predecessors.

Remark 1 Notice that from Definition 3(a), direct links only follow from the leaders to middle level workers, and from middle level workers to terminal workers. The length of any path in a hierarchy is hence bounded above by the height of the hierarchy. Also, links between nodes on the same level are not allowed.

When the partition is obvious from the context, I refer to the adjacency matrix $\mathbf{d}$ as the hierarchy. Here are some examples of hierarchies. 
Example $1\left(\mathcal{T}_{N}\right)$ Figure 6a is a hierarchy with $N=5$, which is the standard team structure. The general team with $N$ members is denoted $\mathcal{T}_{N}$.

Example $2\left(\mathcal{L}_{(1, N-1)}\right)$ Figure 6b is a hierarchy with height 2, one level with one leader and the other level with 4 direct followers. This is the structure explored in Hermalin (1998). The general leading by example hierarchy with one leader and $N-1$ followers is denoted $\mathcal{L}_{(1, N-1)}$.

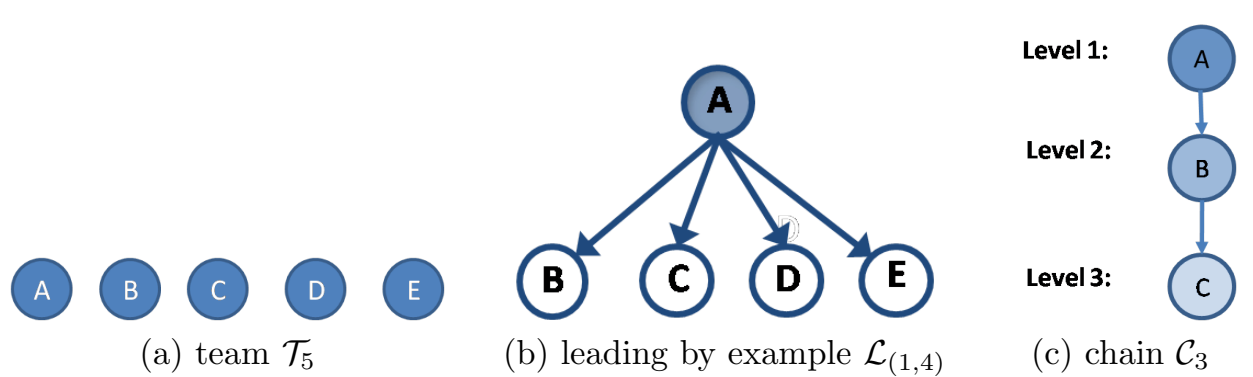

Figure 6: Examples of Hierarchies

Example $3\left(\mathcal{C}_{N}\right)$ Figure $6 c$ is a hierarchy with three levels. Member $B$ follows $A$, but is followed by $C$. The general chain with $N$ workers is denoted $\mathcal{C}_{N}$.

I have analyzed these examples in section 2 for the case $N=3$. A common feature of the above three examples is that a worker has at most one direct predecessor, and hence can make inferences regarding the state only through that predecessor's effort. The equilibrium outcomes are quite different when a worker can draw multiple inferences of the state from efforts of his predecessors. I distinguish these two cases.

Definition 5 A hierarchy $\mathcal{H}$ is called simple if any worker who is not a leader has a unique direct predecessor; $\mathcal{H}$ is called complicated if it is not simple.

In Examples 1-3, hierarchies are all simple. The $V$ structure (Figure 4 is a complicated hierarchy.

Given the payoff defined by the contract $\mathbf{s}$, and the timing defined by the hierarchy $\mathcal{H}$, I can study the equilibrium and make welfare comparisons across different hierarchies as I did in the previous section. 


\section{Simple Hierarchies}

In this section I study the general case of simple hierarchies.

Given an affine-shares contract $\mathbf{s}=\left\{s_{i}, i \in \mathcal{N}\right\} \in \Delta^{N}$ and a simple hierarchy $\mathcal{H}$, define an $h+1$ stage dynamic game $G(\mathbf{s}, \mathcal{H})$ as follows:

- $t=0$, nature chooses $\theta$, which is unknown to all.

- $t=1$, the leaders in $N^{1}$ learn $\theta$ and exert effort simultaneously.

- $t=2$, each member $j \in N^{2}$ observes the effort of his unique direct predecessor $D P^{j}$ (a singleton set in this case), who exerted effort in period $t=1$. Then, members in $N^{2}$ exert effort simultaneously.

- $t=k+1$, each member $l \in N^{k+1}$ observes the effort of his unique direct predecessor $D P^{l}$, who exerted effort in period $t=k$. Then, members in $N^{1+k}$ exert effort simultaneously.

- Payoff is realized and output is divided according to $\mathbf{s}$.

The following theorem fully characterizes the separating equilibrium.

Theorem 4.1 (Equilibrium Characterization) If the hierarchy $\mathcal{H}$ is simple, then there exists a separating equilibrium of $G(\mathbf{s}, \mathcal{H})$ in which the equilibrium efforts $\tilde{x}_{i}(\theta)$ are the solutions to the following system of differential equations:

$$
s_{i} \theta\left(1+\sum_{j \in F^{i}} \frac{\tilde{x}_{j}^{\prime}(\theta)}{\tilde{x}_{i}^{\prime}(\theta)}\right)=c^{\prime}\left(\tilde{x}_{i}(\theta)\right), \quad i=1,2, \cdots, N .
$$

If $F^{i}=\emptyset$, then the summation $\sum_{j \in F^{i}} \frac{\tilde{x}_{j}^{\prime}(\theta)}{\tilde{x}_{i}^{\prime}(\theta)}$ is zero by definition.

This result follows from a standard cost-benefit analysis. If player $i$ deviates by exerting $\Delta x_{i}$ more effort, then that effort will affect the beliefs of all his followers 
by $\Delta \theta \approx \frac{\Delta x_{i}}{\tilde{x}_{i}^{\prime}(\theta)} 9^{9}$ Hence each $j \in F^{i}$ will contribute more by the amount $\tilde{x}_{j}(\theta+\Delta \theta)-$ $\tilde{x}_{j}(\theta) \approx \Delta \theta \cdot \tilde{x}_{j}^{\prime}(\theta)$. Therefore the benefit of this deviation to $i$ is approximately

$$
s_{i} \theta\left(\Delta x_{i}+\sum_{j \in F^{i}} \Delta \theta \cdot x_{j}^{\prime}(\theta)\right) \approx s_{i} \theta\left(1+\sum_{j \in F^{i}} \frac{\tilde{x}_{j}^{\prime}(\theta)}{\tilde{x}_{i}^{\prime}(\theta)}\right) \Delta x_{i}
$$

The cost of this deviation is:

$$
c\left(\tilde{x}_{i}(\theta)+\Delta x_{i}\right)-c\left(\tilde{x}_{i}(\theta)\right) \approx c^{\prime}\left(\tilde{x}_{i}(\theta)\right) \Delta x_{i}
$$

In equilibrium, the benefit equals the cost, which leads to Equation (2).

The equilibrium characterization from Theorem 4.1 has some interesting features. First, the equilibrium effort of player $i$ only depends on his share $s_{i}$ and the equilibrium efforts of his followers. It does not depend on the effort of his predecessors, or on the effort of other workers on the same level as him. Again, this follows from additive separability of the production function. Second, all followers, not just direct followers, affect equilibrium effort. Third, these equations are recursively solvable. Solving for a player's equilibrium effort function requires solving for all of his followers' equilibrium efforts, which requires solving for the equilibrium efforts of the followers' followers, etc.

The term $s_{i} \theta$ comes from $i$ 's share of the output, and the term $s_{i} \theta \sum_{j \in F^{i}} \frac{\tilde{x}_{j}^{\prime}(\theta)}{\tilde{x}_{i}^{\prime}(\theta)}$ comes from $i$ 's signaling incentive and it is always nonnegative. If the signaling term $\sum_{j \in F^{i}} \frac{\tilde{x}_{j}^{\prime}(\theta)}{\tilde{x}_{i}^{\prime}(\theta)}$ vanishes in equation 2, the solutions are just the efforts that workers would expend under symmetric information. With this extra signaling incentive, in equilibrium $i$ must expend more effort. Formally, we have:

Proposition 4.2 The equilibrium effort $\tilde{x}_{i}(\theta)$ of player $i$ characterized in Theorem 4.1 is greater than his effort with symmetric information; that is,

$$
\tilde{x}_{i}(\theta) \geq c^{\prime-1}\left(s_{i} \theta\right) \quad i=1,2, \cdots, N .
$$

Strict inequality holds if $\theta>0$ and $F^{i} \neq \emptyset$.

Proof The weak version is obvious. If $\theta>0$ and $F^{i} \neq \emptyset$, then $\left(1+\sum_{j \in F^{i}} \frac{\tilde{x}_{j}^{\prime}(\theta)}{\tilde{x}_{i}^{\prime}(\theta)}\right)>$ 1 , hence $c^{\prime}\left(\tilde{x}_{i}(\theta)\right)>s_{i} \theta$, or $\tilde{x}_{i}(\theta)>c^{\prime-1}\left(s_{i} \theta\right)$.

\footnotetext{
${ }^{9}$ If $\mathcal{H}$ is simple, a deviation by player $i$ only affects his followers.
} 
Finding closed-form solutions to the above system of equations (2), in general, is infeasible. To get explicit solutions, further restrictions on the disutility functions are needed.

Assumption C: The disutility function is $c(x)=\frac{1}{2} x^{2}$.

Theorem 4.3 Under Assumption $C$, the solutions to equation (2) are $\tilde{x}_{i}(\theta)=$ $k_{i}(\mathbf{s}, \mathcal{H}) \theta$, where $\mathbf{k}(\mathbf{s}, \mathcal{H})=\left\{k_{i}(\mathbf{s}, \mathcal{H}), i \in \mathcal{N}\right\}$ satisfies the following equations:

$$
k_{i}(\mathbf{s}, \mathcal{H})=\frac{s_{i}+\sqrt{s_{i}^{2}+4 s_{i}\left(\sum_{j \in F^{i}} k_{j}(\mathbf{s}, \mathcal{H})\right)}}{2}, \quad i=1,2, \cdots N
$$

Proof For brevity, I write $k_{i}(\mathbf{s}, \mathcal{H})$ as $k_{i}$. If $\tilde{x}_{i}(\theta)=k_{i} \theta$, then $\tilde{x}_{i}^{\prime}(\theta)=k_{i}$, so equation (2) is equivalent to:

$$
s_{i} \theta\left(1+\sum_{j \in F^{i}} \frac{k_{j}}{k_{i}}\right)=c^{\prime}\left(\tilde{x}_{i}(\theta)\right)=\tilde{x}_{i}(\theta)=k_{i} \theta, \quad i=1,2, \cdots, N
$$

Canceling $\theta$ :

$$
s_{i}\left(1+\frac{\sum_{j \in F^{i}} k_{j}}{k_{i}}\right)=k_{i}, \quad i=1,2, \cdots, N
$$

Solving this quadratic equation gives us $k_{i}=\frac{s_{i}+\sqrt{s_{i}^{2}+4 s_{i}\left(\sum_{j \in F^{i}} k_{j}\right)}}{2}$.

Define $g(x, y)=\frac{x+\sqrt{x^{2}+4 x y}}{2}, x>0, y \geq 0$ to be the unique positive solution to

$$
x\left(1+\frac{y}{g(x, y)}\right)=g(x, y) .
$$

See Lemma A.1 in the appendix for some useful properties of $g$. Then equation (3) can be rewritten as:

$$
k_{i}(\mathbf{s}, \mathcal{H})=g\left(s_{i}, \sum_{j \in F^{i}} k_{j}(\mathbf{s}, \mathcal{H})\right), \quad i=1,2, \cdots, N
$$

Theorem 4.3 gives linear solutions for equation (2) under quadratic disutility. The constant $k_{i}$, which quantifies how player $i$ responds to his belief about the 
state in equilibrium, is called $i$ 's responsive coefficient. These coefficients can be calculated by equation 3 recursively:10

From Theorem 4.1 and Theorem 4.3, we see that each player's equilibrium effort depends positively on two components: one is the worker's share $s_{i}$ of the total output, the other is the signaling part depending on the responsive coefficients of $i$ 's followers. For two workers with the same shares, if one worker is the follower of the other, then the signaling incentive of that follower should be weaker than the leader. It is intuitive to guess that the equilibrium effort of the leader should be higher than the follower. This result is formally presented in the following proposition.

Proposition 4.4 If $s_{i}=s_{j}>0$ and $j \in F^{i}$, then $\tilde{x}_{i}(\theta)>\tilde{x}_{j}(\theta)$.

Proof If $j \in F^{i}$, then $F^{j} \subset F^{i}$. Therefore $k_{i}(\mathbf{s}, \mathcal{H})>k_{j}(\mathbf{s}, \mathcal{H})$ by Theorem 4.3 . hence $\tilde{x}_{i}(\theta)>\tilde{x}_{j}(\theta)$.

As a special case, for equal shares, we have:

Corollary 4.5 If $s_{i}=1 / N, \forall i$, then the higher a player is in the hierarchy, the larger the equilibrium effort, and the smaller his equilibrium payoff.

Proof If $s_{i}=s_{j}=1 / N$ and $j \in F^{i}$, then $\tilde{x}_{i}(\theta)>\tilde{x}_{j}(\theta)$ by Proposition 4.4 . therefore $c\left(\tilde{x}_{i}(\theta)\right)>c\left(\tilde{x}_{j}(\theta)\right)$. Then $i$ works harder than $j$, but gets the same share of the output as $j$, so $i$ 's equilibrium payoff must be smaller.

\subsection{Welfare Comparisons for Simple Hierarchies}

In the previous subsection, I solved for the equilibrium efforts for a fixed hierarchy. The aggregate welfare definitely depends on the members' shares, but it also depends crucially on the structure of the hierarchy: how many members are on

\footnotetext{
${ }^{10}$ Here is a simple algorithm to compute all the $\left\{k_{i}\right\}_{i=1}^{N}$ in N steps:

1. Start with terminal workers $j \in N^{h}$. Notice that $k_{j}=s_{j}$ for these workers.

2. Suppose we have computed $k_{j}$ for all workers in $N^{h}, N^{h-1}, \cdots, N^{k}$. Then we can calculate $k_{i}$ for each $i \in N^{k-1}$ using $k_{i}=g\left(s_{i}, \sum_{j \in F^{i}} k_{j}\right)$, since $F^{i} \subset \cup_{k \leq t \leq h} N^{t}$.
} 
each level, how are they connected with each other, how many direct or indirect followers each worker has, and what the followers' shares are.

The aggregate welfare in the equilibrium characterized in Theorem 4.3 is

$$
\begin{aligned}
S W(\mathbf{s}, \mathcal{H}) & =\theta \sum_{i \in \mathcal{N}} k_{i}(\mathbf{s}, \mathcal{H}) \theta-\sum_{i \in \mathcal{N}} \frac{1}{2}\left(k_{i}(\mathbf{s}, \mathcal{H}) \theta\right)^{2} \\
& =\theta^{2} \sum_{i \in \mathcal{N}}\left(k_{i}(\mathbf{s}, \mathcal{H})-\frac{k_{i}(\mathbf{s}, \mathcal{H})^{2}}{2}\right)
\end{aligned}
$$

Clearly, the value of $\theta$ is irrelevant if we want to maximize aggregate welfare with respect to the hierarchy structure and the shares. Equivalently we define $w(\mathbf{s}, \mathcal{H}):=\sum_{i \in \mathcal{N}}\left(k_{i}(\mathbf{s}, \mathcal{H})-\frac{k_{i}(\mathbf{s}, \mathcal{H})^{2}}{2}\right)$. Then the welfare maximization problem can be written as

$$
\max _{\mathbf{s}, \mathcal{H}} w(\mathbf{s}, \mathcal{H}) \quad \text { subject to } \sum_{i \in \mathcal{N}} s_{i}=1, s_{i} \geq 0 .
$$

This program can be decomposed into two steps:

1. For a fixed hierarchy $\mathcal{H}$, find the optimal shares $\mathbf{s}^{*}(\mathcal{H})$ by solving the following problem:

$$
\max _{\mathbf{s} \geq \mathbf{0}, \sum_{i \in \mathcal{N}} s_{i}=1} w(\mathbf{s}, \mathcal{H})=\sum_{i \in \mathcal{N}}\left(k_{i}-\frac{k_{i}^{2}}{2}\right)
$$

We define $\bar{w}(\mathcal{H}):=w\left(\mathbf{s}^{*}(\mathcal{H}), \mathcal{H}\right)$ as the maximum value above.

2. Maximize over different simple hierarchies with optimal shares $\mathbf{s}^{*}(\mathcal{H})$ :

$$
\max _{\mathcal{H}} w\left(\mathbf{s}^{*}(\mathcal{H}), \mathcal{H}\right)=\max _{\mathcal{H}} \bar{w}(\mathcal{H})
$$

Below is the main result of this paper.

Theorem 4.6 (Optimal Simple Hierarchy) The chain is the optimal simple hierarchy, i.e.,

$$
\bar{w}(\mathcal{H})=\max _{\mathbf{s} \geq \mathbf{0}, \sum_{i \in \mathcal{N}} s_{i}=1} w(\mathbf{s}, \mathcal{H}) \leq \bar{w}\left(\mathcal{C}_{N}\right)=\max _{\mathbf{s} \geq \mathbf{0}, \sum_{i \in \mathcal{N}} s_{i}=1} w\left(\mathbf{s}, \mathcal{C}_{N}\right)
$$


The proof is shown in a sequence of steps.

First, to make two hierarchies comparable, we transform any hierarchy $\mathcal{H}$ into a chain, and compare the equilibrium efforts under these two hierarchies using the same share profile. Each transformation is determined by a permutation $\sigma$ of $\mathcal{N}$ by assigning member $\sigma(i)$ to the $i$ th level on a chain, $i=1, \cdots, N$. For a fixed hierarchy $\mathcal{H}$, we look for a special permutation $\sigma$ satisfying the following condition:

Condition OP: If $j$ is $i$ 's follower under $\mathcal{H}$, then $\sigma(i)<\sigma(j)$.

The existence of such a permutation is shown in the following lemma. 11

Lemma 4.7 For any hierarchy $\mathcal{H}$, there exists a permutation $\sigma$ satisfying Condition $O P$.

Proof For each $i$, let $f_{i}=\# F^{i}$ be the number of followers of $i$ under $\mathcal{H}$. We can enumerate the numbers in $\mathcal{N}$ by decreasing order of $f_{i}$ (take any order if $f_{i}=f_{j}$ for $i \neq j$ ). We define the permutation $\sigma$ by mapping each $i$ to its place under this enumeration. Then, if $j \in F^{i}, j$ 's follower is also $i$ 's follower, hence $F^{j}$ is a strict subset of $F^{i}$. Therefore $f_{i}>f_{j}$, so $i$ must come earlier in $\sigma$ than $j$, equivalently, $\sigma(i)<\sigma(j)$.

With the permutation $\sigma$ given in the above lemma, we construct the chain $\mathcal{C}_{(\mathcal{N})}^{\sigma}$ by assigning member $\sigma(i)$ to the $i$ th level, $i=1, \cdots, N$. We call this the $\mathbf{C}$ transformation, because this procedure transforms any hierarchy into a chain. Given a share profile $\mathbf{s}$ for $\mathcal{H}$, we assign the same share $s_{i}$ to member $i$ in the chain $\mathcal{C}_{(\mathcal{N})}^{\sigma}$. Condition OP implies that this transformation has some nice properties.

Lemma 4.8 The C-transformation given by a permutation $\sigma$ satisfying condition OP has the following properties.

1. For fixed shares $\mathbf{s}$, any member's equilibrium effort is weakly higher in $\mathcal{C}_{(\mathcal{N})}^{\sigma}$ than in $\mathcal{H}$, that is,

$$
k_{i}\left(\mathbf{s}, \mathcal{C}_{(\mathcal{N})}^{\sigma}\right) \geq k_{i}(\mathbf{s}, \mathcal{H})
$$

2. For fixed shares $\mathbf{s}$, we can find another share profile $\tilde{\mathbf{s}}$ such that $\tilde{\mathbf{s}} \leq \mathbf{s}$ but

$$
k_{i}\left(\tilde{\mathbf{s}}, \mathcal{C}_{(\mathcal{N})}^{\sigma}\right)=k_{i}(\mathbf{s}, \mathcal{H})
$$

\footnotetext{
${ }^{11}$ There are multiple permutations satisfying Condition OP. We just need one.
} 
Therefore, $w\left(\tilde{\mathbf{s}}, \mathcal{C}_{(\mathcal{N})}^{\sigma}\right)=w(\mathbf{s}, \mathcal{H})$.

The C-transformation preserves the subordination relation between members. If $i$ is a predecessor of $j$ in $\mathcal{H}$, then $i$ is $j$ 's predecessor in $\mathcal{C}_{(\mathcal{N})}^{\sigma}$. For some players, however, the set of followers may be strictly larger in $\mathcal{C}_{(\mathcal{N})}^{\sigma}$. Each worker's equilibrium effort depends on his own share and the sum of equilibrium efforts of all his followers. Since the shares are the same under the two hierarchies, each member will have larger incentive to signal when he has more followers in $\mathcal{C}_{(\mathcal{N})}^{\sigma}$, therefore his equilibrium effort is weakly higher. Moreover we can reduce his share suitably to make his equilibrium effort equal under two hierarchies. Therefore for any hierarchy $\mathcal{H}$ with a share profile $\mathbf{s}$, the chain $\mathcal{C}_{(\mathcal{N})}^{\sigma}$ can generate the same welfare using a share profile $\tilde{\mathbf{s}}$, which uses less total shares than $\mathbf{s}$. But $\tilde{\mathbf{s}}$ in general does not belong to $\Delta^{N}$ even if the initial share profile $\mathbf{s}$ is in $\Delta^{N}$.

Next we show that if we distribute the the extra shares optimally to the team members in the chain, we can generate even higher welfare. Actually we show this result for any hierarchy, not just the chain.

Formally, for each fixed $\mathcal{H}$ and $t \in(0,1]$, define

$$
\begin{aligned}
\phi(t, \mathcal{H}):=\quad & \max _{\mathbf{s}} w(\mathbf{s}, \mathcal{H}) \\
& \text { s.t: } \sum_{i \in \mathcal{N}} s_{i}=t, s_{i} \geq 0
\end{aligned}
$$

as the maximal achievable welfare under $\mathcal{H}$ with the constraint that the total shares sum up to $t$. In particular when $t=1, \phi(1, \mathcal{H})=\bar{w}(\mathcal{H})$. In the appendix, we show that increasing the total constraint on shares improves welfare whenever total shares are less than 1.

Theorem 4.9 For any simple hierarchy $\mathcal{H}$, if $1 \geq t_{1}>t_{2} \geq 0$, then $\phi\left(t_{1}, \mathcal{H}\right)>$ $\phi\left(t_{2}, \mathcal{H}\right)$.

Using Lemma 4.8 and Theorem 4.9 , we show that the chain structure is optimal whenever the total shares are less than 1.

Proposition 4.10 For any simple hierachy $\mathcal{H}, \phi(t, \mathcal{H}) \leq \phi\left(t, \mathcal{C}_{N}\right), \forall t \in(0,1]$.

Proof For any $t \in(0,1]$, suppose $\mathbf{s}$ is optimal, i.e., $\phi(t, \mathcal{H})=w(\mathbf{s}, \mathcal{H})$. Then from Lemma 4.8 , we can find a welfare equivalent $\tilde{\mathbf{s}}$ in the chain $C_{(N)}^{\sigma}$, but use less total 
share, i.e, $w(\mathbf{s}, \mathcal{H})=w\left(\tilde{s}, \mathcal{C}_{(\mathcal{N})}^{\sigma}\right),|\tilde{\mathbf{s}}| \leq|\mathbf{s}|=t$. By Theorem 4.9, extra shares always yield greater welfare if we adjust the shares optimally. Formally, we have:

$$
\begin{aligned}
\phi(t, \mathcal{H}) & =w(\mathbf{s}, \mathcal{H})=w\left(\tilde{s}, \mathcal{C}_{(\mathcal{N})}^{\sigma}\right) \\
& \leq \max _{\mathbf{s} \geq 0,|s|=|\tilde{\mathbf{s}}|} w\left(s, \mathcal{C}_{(\mathcal{N})}^{\sigma}\right)=\phi\left(|\tilde{\mathbf{s}}|, \mathcal{C}_{(\mathcal{N})}^{\sigma}\right) \leq \phi\left(t, \mathcal{C}_{(\mathcal{N})}^{\sigma}\right)
\end{aligned}
$$

The first inequality holds since $\tilde{\mathbf{s}}$ is not necessarily optimal. The second inequality follows from Theorem 4.9 since $|\tilde{\mathbf{s}}| \leq|\mathbf{s}|=t$. Note that both $\mathcal{C}_{(\mathcal{N})}^{\sigma}$ and $\mathcal{C}_{N}$ represent the same hierarchy: the chain of length $N$. Therefore, $\phi\left(t, \mathcal{C}_{(\mathcal{N})}^{\sigma}\right)=\phi\left(t, \mathcal{C}_{N}\right) \cdot \sqrt{12}$ Thus, we finish the proof of Proposition 4.10.

Proposition 4.10 immediately implies Theorem 4.6 by setting $t=1$.

The optimality of the chain comes from three observations. First, the chain gives every member the maximal signaling incentives. Note that motivating efforts alone is not always welfare improving, as the welfare function is declining after effort exceeds first-best level. Second, we can use fewer shares to provide incentives to the workers in the chain to generate the same levels of efforts than in any other hierarchy. Third, extra shares, if distributed optimally among the team, improve welfare.

Remark 2 If the main program is to maximize equilibrium output or equivalently $\sum_{i \in \mathcal{N}} k_{i}(\mathbf{s}, \mathcal{H})$ rather than equilibrium welfare, then the chain is still optimal, since each worker's equilibrium effort is weakly higher after C-transformation (see Lemma 4.8).

Remark 3 If the social welfare generated by the first hierarchy is higher than the second one, we can use lump-sum transfers $t_{i}, i=1, \cdots n$, which won't affect incentives of workers, to make sure that every worker's equilibrium payoff in the first hierarchy is higher than that in the second hierarchy. Therefore, the chain structure will make all workers better off.

\footnotetext{
${ }^{12}$ This result does not hold if the team is not homogeneous either because different workers have different disutility functions, or because different workers value the project differently.
} 


\subsection{The Chain $\mathcal{C}_{N}$}

Given the optimality of the chain, we next study the optimal sharing rule and equilibrium effort levels in the chain. We give a detailed analysis of the chain in this subsection.

Consider the chain $\mathcal{C}_{N}$. We denote by $i$ the unique worker on level $i, i=$ $1, \cdots, N$. Let $s_{i}$ be worker $i$ ' share. Then by Theorem 4.3, we have the following expression for $k_{i}\left(\mathbf{s}, \mathcal{C}_{N}\right)$ :

$$
\begin{aligned}
k_{N}\left(\mathbf{s}, \mathcal{C}_{N}\right) & =g\left(s_{N}, 0\right)=s_{N} \\
k_{N-1}\left(\mathbf{s}, \mathcal{C}_{N}\right) & =g\left(s_{N-1}, k_{N}\left(\mathbf{s}, \mathcal{C}_{N}\right)\right) \\
& \vdots \\
k_{1}\left(\mathbf{s}, \mathcal{C}_{N}\right) & =g\left(s_{1}, \sum_{j=2}^{N} k_{j}\left(\mathbf{s}, \mathcal{C}_{N}\right)\right)
\end{aligned}
$$

\subsubsection{Equal shares}

To get quantitative results about the strength of sequential signaling effects - and also their limitations - here we calculate the welfare function for equal share $\mathbf{s}^{e q}$, that is, $s_{i}=\frac{1}{N}, \forall i$. Equal shares corresponds to the case of public good provision or committee service, as each team member has roughly the same stake in the project.

First, we have the following estimates regarding responsive coefficients:

Lemma 4.11 For equal shares $\mathbf{s}^{e q}$ in the chain $\mathcal{C}_{N}$, we have

$$
\frac{1}{2 N}<k_{i}\left(\mathbf{s}^{e q}, \mathcal{C}_{N}\right)-k_{i+1}\left(\mathbf{s}^{e q}, \mathcal{C}_{N}\right)<\frac{1}{N} \quad i=1, \cdots, N-1
$$

Hence:

$$
\frac{N+1-i}{2 N}<k_{i}\left(\mathbf{s}^{e q}, \mathcal{C}_{N}\right)<\frac{N+1-i}{N} \quad i=1, \cdots, N-1
$$

In particular, $k_{i}\left(\mathbf{s}^{e q}, \mathcal{C}_{N}\right)<1, \forall i$.

With these bounds, we can estimate the welfare $S W\left(\mathbf{s}^{e q}, \mathcal{C}_{N}\right)$.

Proposition 4.12 The aggregate welfare for $\mathcal{C}_{N}$ with equal shares, $S W\left(\mathbf{s}^{e q}, \mathcal{C}_{N}\right)$, satisfies:

$$
\frac{5}{24} N \theta^{2} \leq S W\left(\mathbf{s}^{e q}, \mathcal{C}_{N}\right) \leq\left(\frac{1}{3} N+\frac{1}{4}\right) \theta^{2}
$$


The first-best aggregate welfare is $\frac{1}{2} N \theta^{2}$, which is, of course, infeasible here. Also, the free-riding problem is more severe in larger teams as each member's share is smaller. However, Proposition 4.12 shows that the signaling incentives in the team are strong enough to yield at least $\frac{5}{12} \approx 42 \%$ of the first-best welfare with equal shares. Optimal shares potentially could do better.

From Lemma 4.11, we see that for the equal share rule, all the $k_{i}$ are less the first-best. From Example 4 (below), however, we see that $k_{i}$ could be greater than the first-best under some shares. So, we look for a sufficient condition on $\mathbf{s}$ to guarantee that $k_{i}\left(\mathbf{s}, \mathcal{C}_{N}\right) \leq 1$. The following proposition is one result along this line.

Proposition 4.13 If the shares $\mathbf{s}$ for the chain are monotonic, so that $0<s_{1} \leq$ $s_{2} \cdots \leq s_{N}$, then $k_{i}\left(\mathbf{s}, \mathcal{C}_{N}\right)<1, \forall i$.

The assumption of this proposition is quite general. Equal shares satisfy this condition. By continuity, this conclusion still holds for shares $s$ sufficiently close to $s^{e q}$ or any monotonic shares. Note that $\mathbf{s}^{\prime}=(0.8,0.1,0.1)$ in Example 4 is not monotonic, and indeed $k_{A}=1.007$ is greater than 1 for $\mathbf{s}^{\prime}$.

\subsubsection{Optimal shares}

In this subsection, we characterize the optimal shares for the chain with $N$ workers. First, we start with an example with three workers.

Example 4 Table 1 lists the equilibrium efforts and welfare with different share rules, where $A$ is the leader, $C$ is the terminal worker, and $B$ is the middle worker. $\mathbf{s}^{e q}$ are the equal shares, and $\mathbf{s}^{*}$ are the optimal shares.

\begin{tabular}{|c|c|c|}
\hline $\mathbf{s}=\left(s_{A}, s_{B}, s_{C}\right)$ & $\mathbf{k}=\left(k_{A}, k_{B}, k_{C}\right)$ & welfare $w\left(\mathbf{s}, \mathcal{C}_{3}\right)$ \\
\hline $\mathbf{s}^{\prime}=(0.8,0.1,0.1)$ & $(\mathbf{1 . 0 0 7 8}, 0.1618,0.1000)$ & 0.7437 \\
\hline $\mathbf{s}^{\prime \prime}=(0.75,0.1,0.15)$ & $(0.9994,0.1823,0.1500)$ & 0.8044 \\
\hline $\mathbf{s}^{e q}=(0.3333,0.3333,0.3333)$ & $(0.7312,0.5393,0.3333)$ & 1.1355 \\
\hline $\mathbf{s}^{*}=(0.1997,0.2668,0.5335)$ & $(0.5721,0.5335,0.5335)$ & 1.1909 \\
\hline
\end{tabular}

Table 1: $\mathcal{C}_{3}$ with different shares 
There are a few points worth noting. Because $k_{A}\left(\mathbf{s}^{\prime}, \mathcal{C}_{3}\right)>1$, A's equilibrium effort is actually higher than first-best level. Transferring a small share from A to C, as shown in $\mathbf{s}^{\prime \prime}$, is welfare improving, because it mitigates A's overly strong signaling incentive in $\mathbf{s}^{\prime}$ and gives $\mathrm{C}$ more shares so that $\mathrm{C}$ will expend higher effort. For equal shares $\mathbf{s}^{e q}$, efforts are monotonic, but still below first-best levels (Lemma 4.11). But equal shares are not optimal, i.e., $\mathbf{s}^{e q} \neq \mathbf{s}^{*}$. Moreover, for optimal shares, $s_{A}^{*}<s_{B}^{*}<s_{C}^{*}$, but $k_{A}^{*}>k_{B}^{*}=k_{C}^{*}$. The worker higher in the hierarchy actually has a smaller share, but works harder due to stronger signaling incentives from his followers. We will see that this is the general pattern for the chain of any length.

Equal shares are in general not optimal. The effort of workers on higher levels is too high compared with workers on lower levels. Now we turn to the question of how to find the optimal shares. Unfortunately, the expressions for $k_{i}$ are recursive and the exact expressions involving $\left\{s_{i}\right\}$ are quite complicated. Therefore the Lagrange multiplier approach to maximize $w\left(\mathbf{s}, \mathcal{C}_{N}\right)$ with constraint $\mathbf{s} \in \Delta^{N}$ is not quite informative. Nevertheless, we use a variation argument to show that optimal shares $\mathbf{s}^{*}$ and the corresponding responsive coefficients $k_{i}^{*}=k_{i}\left(\mathbf{s}^{*}, \mathcal{C}_{N}\right)$ satisfy the following conditions:

Theorem 4.14 The optimal shares $\mathbf{s}^{*}$ for the chain satisfy

$$
\frac{1}{2}<\frac{s_{i}^{*}}{s_{i+1}^{*}}<1, \quad \forall i=1,2, \cdots, N-2, \quad \text { and } \quad \frac{s_{N-1}^{*}}{s_{N}^{*}}=\frac{1}{2} .
$$

Moreover, the $k_{i}^{*}$ s satisfy $1>k_{1}^{*}>k_{2}^{*}>\cdots>k_{N-1}^{*}=k_{N}^{*}>0$.

As a consequence, we get a chain of inequalities:

$$
0<s_{1}^{*}<s_{2}^{*}<\cdots<s_{N}^{*}=k_{N-1}^{*}=k_{N}^{*}<\cdots<k_{2}^{*}<k_{1}^{*}<1 .
$$

All workers are symmetric ex ante, but we do not want to distribute the shares equally to them as different workers have different signaling incentives that vary with the their positions on the chain. Under optimal shares in the chain, a worker has stronger signaling incentives than his followers, although he gets a smaller share of the output. 


\section{Complicated Hierarchies}

Although simple hierarchies are the structures typically observed in organizations, complicated hierarchies exist as well. Sometimes a worker may communicate with multiple bosses. I explore complicated hierarchies in this section.

For a complicated hierarchy, we can define the dynamic signaling game as before, except that now a worker can potentially observe efforts from multiple direct predecessors. After adopting the pessimistic belief assumption as I did for the $V$ structure with two leaders, the equilibrium characterization is quite similar to the case of simple hierarchies.

Theorem 5.1 If $\mathcal{H}$ is complicated, then there exists a separating equilibrium of $G(\mathbf{s}, \mathcal{H})$ in which the equilibrium efforts are $\tilde{x}_{i}(\theta)=k_{i}(\mathbf{s}, \mathcal{H}) \theta$, while $k_{i}(\mathbf{s}, \mathcal{H})$ is given recursively by

$$
k_{i}(\mathbf{s}, \mathcal{H})=g\left(s_{i}, \sum_{j \in F^{i}} k_{j}(\mathbf{s}, \mathcal{H})\right), \quad i=1,2, \cdots N .
$$

Qualitatively, Theorem 5.1 looks exactly the same as Theorem 4.3. There are some major differences. First, we need the pessimistic belief assumption in complicated hierarchies, while we did not need any assumption on the belief functions for simple hierarchies. Second, there are multiple equilibria even with the pessimistic belief assumption as we have seen in the $V$ structure (Section 2). Uniqueness of the equilibrium is not guaranteed.

From now on, we focus on the equilibrium identified by Theorem 5.1 for both simple and complicated hierarchies. Given the similarity between Theorem 5.1 and Theorem 4.3, we can show that the results proved for simple hierarchies, like Proposition 4.4, Lemma 4.8, and Theorem 4.9, also hold for complicated hierarchies. I will directly use them without proof.

\subsection{Welfare Improving Operations}

In section 4.1, we have shown that the chain structure is optimal among simple hierarchies. Next we want to show that the chain structure is optimal among all hierarchies, simple or complicated. To achieve this goal, we explore two welfareimproving operations on hierarchies. 
Definition 6 For fixed hierarchy $\mathcal{H}=(\mathbf{d}, \mathcal{P})$, let $\mathcal{H}+i j$ be the hierarchy formed from $\mathcal{H}$ by adding one direct link from $i$ to $j$ in $\mathbf{d}$.

Note that in the definition $i$ and $j$ should not be linked in $\mathcal{H}$, and $j$ should lie on the next level from $i$, otherwise $\mathcal{H}+i j$ is not a hierarchy according to Definition 3. Obviously, $\mathcal{H}+i j$ is not simple even if $\mathcal{H}$ is. Adding links enlarges the sets of followers, hence pushes workers' effort higher by Theorem 5.1. Formally:

Proposition 5.2 For fixed share profile $\mathbf{s}, k_{l}(\mathbf{s}, \mathcal{H}) \leq k_{l}(\mathbf{s}, \mathcal{H}+i j), \forall l \in N$.

From Proposition 5.2, it is intuitive to guess that for fixed share profile $\mathbf{s}$, adding links will increase welfare: $w(\mathbf{s}, \mathcal{H}) \leq w(\mathbf{s}, \mathcal{H}+i j)$. We give a counterexample in the Appendix to show that this naive argument is wrong. The reason is that some players' effort is already too high under strong signaling incentives, and motivating higher effort for those workers will actually decrease welfare. Instead, once we adjust the share optimally, we can show that adding links increases welfare.

Theorem 5.3 For every fixed $t \in(0,1], \phi(t, \mathcal{H}) \leq \phi(t, \mathcal{H}+i j)$. In particular, when $t=1, \bar{w}(\mathcal{H}) \leq \bar{w}(\mathcal{H}+i j)$.

Definition 7 A hierarchy $\mathcal{H}$ is maximal if any member in $N^{k}$ is linked to any member in $N^{k+1}$, for $k=1,2, \cdots, h-1$.

Adding links (with suitable adjustment of shares) means improving welfare, therefore we should link all the unlinked workers in adjacent levels (if the partition is fixed). In the end, we construct a maximal hierarchy, i.e, a multi-partition graph. Thus, we have the following corollary.

Corollary 5.4 For hierarchies with $N$ workers, the optimal hierarchy is a multipartition graph if the number of workers on each level is fixed.

For a maximal hierarchy, workers within each level are symmetric. As a special case, for leading by example $\mathcal{L}_{(1, N-1)}$, Hermalin (1998) shows that all $N-1$ followers should get the same shares under optimal affine linear contracts. The following proposition shows that this holds for general maximal hierarchies. 
Proposition 5.5 For maximal hierarchies under optimal shares, members on the same level are assigned the same shares, hence work equally hard in equilibrium. ${ }^{13}$

We still do not know exactly what the optimal shares are for any maximal hierarchy. Nevertheless, these results show that we can reduce the number of variables from $N$, the total number of workers, to $h$, the height of the hierarchy.

Starting from a multi-partition graph, we can split one level into multiple levels. Figure 7 explains such a procedure. We claim that this procedure also improves welfare after adjusting shares optimally.
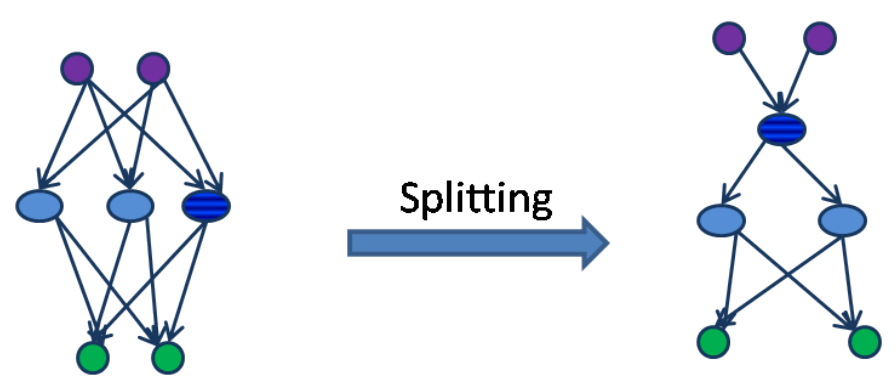

Figure 7: Splitting

Suppose $\mathcal{H}$ is a maximal hierarchy defined by the ordered partition $\mathcal{P}=$ $\left\{N^{1}, \cdots, N^{h}\right\}$ of $\mathcal{N}$. Suppose $\left|N_{k}\right| \geq 2$ for some $k$. Pick $i \in N^{k}$, and let $\mathcal{H}^{\prime}$ be the maximal hierarchy defined by the ordered partition $\mathcal{P}^{\prime}=\left\{N^{1}, \cdots, N^{k-1},\{i\}\right.$, $\left.N^{k} \backslash\{i\}, \cdots, N^{h}\right\}$.

Proposition 5.6 For fixed $t \in(0,1]$, we have:

$$
\phi(t, \mathcal{H})<\phi\left(t, \mathcal{H}^{\prime}\right)
$$

In particular, for $t=1$, we have $\bar{w}(\mathcal{H})<\bar{w}\left(\mathcal{H}^{\prime}\right)$. The new hierarchy constructed by splitting is more efficient than the original one.

By repeated splitting until we have a partition that we cannot split at any level, eventually, we get the chain. Here is a map presenting the procedures to move from any hierarchy to the chain:

$$
\text { hierarchy } \mathcal{H} \stackrel{\text { add links }}{\Longrightarrow} \text { maximal hierarchy } \overbrace{\Longrightarrow}^{\text {splitting }} \text { chain }
$$

\footnotetext{
${ }^{13}$ This result relies on $c(x)=\frac{1}{2} x^{2}$. It may not hold for other cost functions.
} 
Each step is welfare improving, which shows the optimality of the chain structure.

Theorem 5.7 Among all hierarchies, the chain structure is optimal. ${ }^{14}$

This theorem is the counterpart of Theorem 4.6 for simple hierarchies.

\section{Extensions}

\subsection{Who will be a leader?}

Previously, we have only compared the aggregate welfare of the whole team across different hierarchies. In this section, we study an individual's incentive in various hierarchies. As an example, with equal shares, leaders contribute more to the common project. Why should they do that? Who would ever want to a leader? To answer this question, first we review some results from section 2 .

Figure 8 compares the equilibrium payoffs of three workers in various hierarchies with equal share $\mathbf{s}^{e q}$. The number to the right of each node is the payoff of that node when $\theta=1$. SW denotes welfare when $\theta=1$.

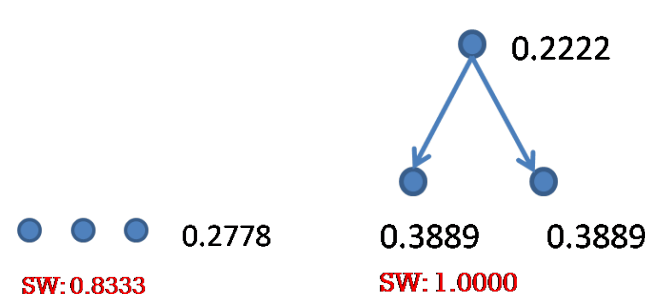

(a) Team $\mathcal{T}_{3}$

(b) Lead by Example $\Lambda$

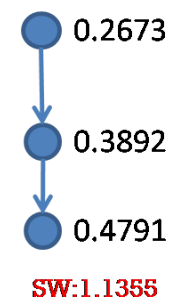

(c) Chain $\mathcal{C}_{3}$

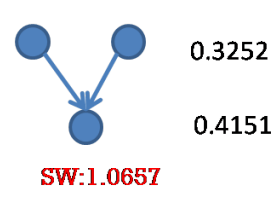

(d) $V$ structure

Figure 8: Individual payoffs with different hierarchies

A general fact illustrated in the figure is that a leader's equilibrium payoff is actually lower than her followers. This is consistent with Corollary 4.5. Another

\footnotetext{
${ }^{14}$ This result depends crucially on the fact that we are picking the equilibrium given in Theorem 5.1 for complicated hierarchies. Using other belief functions or picking other equilibria may reverse the ranking. See the discussion in the appendix about belief functions with the $\mathrm{V}$ structure. We do not have this problem when restricting attention to simple hierarchies.
} 
interesting observation is to compare the payoffs of the leader under the first three hierarchies. The leader's payoff in the $\Lambda$ structure, 0.2222 , is lower than he gets in the team structure, 0.2778. But the leader's payoff is higher in the chain, as the middle player is now contributing more to the project. Meanwhile, given the fact that a lump-sum transfer could be used to adjust individual payoffs without affecting the incentives, thus equilibrium efforts, of workers, it is reasonable to concentrate only on comparisons of aggregate welfare.

\subsection{Limited Height}

The previous analysis has shown that the chain is optimal among all hierarchies, but the height of the chain is too large to be realistic when $N$ is large. Here we search for optimal hierarchies satisfying more realistic conditions, such as a constraint on the number of levels, being simple, and having a single leader. Formally, define

$$
\mathcal{M}^{s}(N, K, 1)=\{\mathcal{H} \mid \mathcal{H} \text { is simple, has height } K \text { and one leader }\}
$$

Here we look for the optimal hierarchy in $\mathcal{M}^{s}(N, K, 1){ }^{15}$

For $K=2$, the program is trivial, since the only hierarchy satisfying all of the three conditions is leading by example $\mathcal{L}_{(1, N-1)}$. The optimal sharing rule for this structure is completely solved in Hermalin (1998) for any $N$.

For $K=3$, the problem gets tricky. For general $N \geq 3, K<N, \mathcal{M}^{s}(N, K, 1)$ is not a singleton. There could be a different number of middle managers, and different groups of followers for each middle manager.

For example, if $N=1+2 p$ is odd and $p \geq 2$, Figure 9 presents two hierarchies $\mathcal{H}^{1}$ and $\mathcal{H}^{2}$ in $\mathcal{M}^{s}(N, 3,1)$. For $\mathcal{H}^{1}$, there are $p$ managers, and each has only one follower; for $\mathcal{H}^{2}$, there are only 2 managers, and each has $p$ followers.

Proposition 6.1 Hierarchy $\mathcal{H}^{1}$ is more efficient than $\mathcal{H}^{2}$ :

$$
\bar{w}\left(\mathcal{H}^{1}\right)=\max _{s \in \Delta^{N}} w\left(\mathbf{s}, \mathcal{H}^{1}\right)>\max _{s \in \Delta^{N}} w\left(\mathbf{s}, \mathcal{H}^{2}\right)=\bar{w}\left(\mathcal{H}^{2}\right)
$$

\footnotetext{
${ }^{15}$ Equivalently, we could define the set $\mathcal{M}^{s}(N, K, 1)$ by including all the hierarchies with height at most $K$. The optimal element would be the same. The reason is that the constraint on height must bind for the optimal hierarchy in $\mathcal{M}^{s}(N, K, 1)$, since it must use the maximum height, i.e, $K$ in this case.
} 


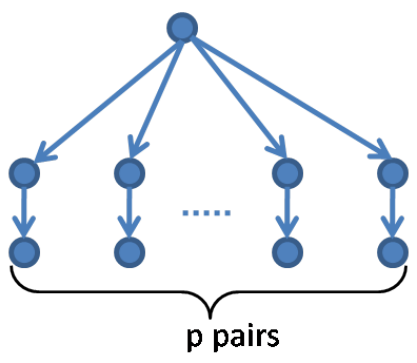

(a) $\mathcal{H}^{1}$

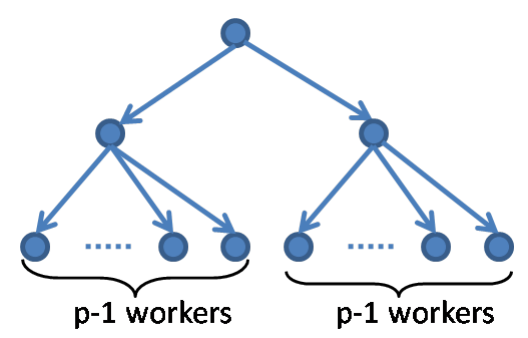

(b) $\mathcal{H}^{2}$

Figure 9: two hierarchical structures in $\mathcal{M}^{s}(N, 3,1)$

The intuition behind this proposition is as follows. Suppose share profile $\mathbf{s}$ is optimal for $\mathcal{H}^{2}$ and let $l$ be the share of the leader, L. Then we can construct a profile $\mathbf{s}^{\prime}$ for $\mathcal{H}^{1}$ as follows: L still gets $l$, while each manager gets $m=\frac{1}{3} \frac{1-l}{p}$ and every terminal worker gets $f=\frac{2}{3} \frac{1-l}{p}$. Note that $g(m, f)=g\left(\frac{1}{2} f, f\right)=f$, so all workers in $\mathcal{H}^{1}$ except $\mathrm{L}$ exert the same equilibrium effort. The sum of responsive coefficients for those $2 p$ workers is

$$
p(g(m, f)+f)=2 p f=\frac{4}{3}(1-l)
$$

We claim (with proof given in the appendix) that the sum of the responsive coefficients of all workers in $\mathcal{H}^{2}$ except the leader $\mathrm{L}$ under the contract $\mathbf{s}$ is less than $\frac{4}{3}(1-l)$, i.e,

$$
\frac{4}{3}(1-l) \geq \sum_{j \neq L} k_{j}\left(\mathbf{s}, \mathcal{H}^{2}\right)
$$

or

$$
f=\frac{2}{3} \frac{1-l}{p} \geq \frac{\sum_{j \neq L} k_{j}\left(\mathbf{s}, \mathcal{H}^{2}\right)}{2 p} .
$$

Note $\eta(k):=k-\frac{1}{2} k^{2}$ is concave in $k$ and increasing if $k<1$, so by Jensen's inequality,

$$
\begin{aligned}
\sum_{j \neq L} \eta\left(k_{j}\left(\mathbf{s}, \mathcal{H}^{2}\right)\right) & =2 p\left(\sum_{j \neq L} \frac{1}{2 p} \eta\left(k_{j}\left(\mathbf{s}, \mathcal{H}^{2}\right)\right)\right) \\
& \leq 2 p \times \eta\left(\frac{\sum_{j \neq L} k_{j}\left(\mathbf{s}, \mathcal{H}^{2}\right)}{2 p}\right) \leq 2 p \times \eta(f)
\end{aligned}
$$


The last inequality holds because $\frac{\sum_{j \neq L} k_{j}\left(\mathbf{s}, \mathcal{H}^{2}\right)}{2 p} \leq f=\frac{2}{3} \frac{1-l}{p}<\frac{2}{3} \frac{1}{p}<1$. This shows the contribution to welfare by all workers except $\mathrm{L}$ is higher in $\mathcal{H}^{1}$ than in $\mathcal{H}^{2}$. The leader's incentive is higher in $\mathcal{H}^{1}$, because her share under the two cases is the same but the sum of the responsive coefficients of her followers is higher in $\mathcal{H}^{1}$ by equation 11 . The old trick applies. We reduce the share of $\mathrm{L}$ by $\Delta>0$ to make her incentive equal and then apply Theorem 4.9 to finish the proof.

The same argument can be used to show that hierarchy $\mathcal{H}^{1}$ is not only more efficient than $\mathcal{H}^{2}$, but also more efficient than any other hierarchy in $\mathcal{M}^{s}(N, 3,1)$.

Proposition 6.2 If $N=1+2 p$ is odd, $p \geq 2$, and $K=3$, then hierarchy $\mathcal{H}^{1}$ is the most efficient in $\mathcal{M}^{s}(N, K, 1)$. That is,

$$
\bar{w}\left(\mathcal{H}^{1}\right)>\bar{w}(\mathcal{H}), \quad \forall \mathcal{H} \in \mathcal{M}^{s}(N, K, 1), \quad \mathcal{H} \neq \mathcal{H}^{1}
$$

\subsection{Endogenous Information Acquisition}

Previously, we have assumed that leaders are endowed with information, How do the leaders get the information in the first place? Presumably, the leaders exert costly research effort, such as sampling, running regressions, or consulting experts, to get more accurate information about the state. In this section, we study endogenous information acquisition in hierarchies.

For simplicity, assume $\mathcal{H}$ is simple, and there is a unique leader, $L$. She is the only one who will acquire information. To study this extension, insert one more stage between $t=0$ (nature chooses $\theta$ ) and $t=1$ (the leader expends her effort $\left.x_{L}\right)$ in the game $G(\mathbf{s}, \mathcal{H})$ :

- $t=0.5$, the leader $\mathrm{L}$ exerts research effort $I \in \mathcal{I}=\left[I_{0}, I_{1}\right]$ and gets a signal $s$.

For each information structure $I, \theta^{I} \sim E^{I}\{\theta \mid s\}$ is the posterior. Let $F^{I}$ be the C.D.F of $\theta^{I}$. Furthermore we assume:

1. The support of $\theta^{I}$ is $\Theta$ for any $I \in \mathcal{I}$.

2. The utility of the leader is additively separable in both research effort and productive effort. 
3. For $I<I^{\prime}, \theta^{I^{\prime}}$ is a mean-preserving spread (MPS) of $\theta^{I}{ }^{16}$

Condition 2 guarantees that the leader's research effort does not affect his signaling incentives. Risk-neutrality of workers and Condition 1 implies that the equilibrium characterization still applies, except that now we have to replace the state by the leader's point estimate in Theorem 4.3. Condition 3 means that the label of the information structure preserves the informativeness of the signal, that is, the higher is $I$, the more spread is the distribution of $\theta^{I}$.

If research effort is verifiable (so it can serve as a contract contingency), then we only need to maximize expected social welfare of information minus the cost of research effort:

$$
\mathcal{U}(\mathcal{H}):=\max _{I \in \mathcal{I}} \max _{\mathbf{s} \in \Delta^{N}}\left(\int_{\Theta} w(\mathbf{s}, \mathcal{H}) \theta^{2} d F^{I}(\theta)-r(I)\right)
$$

Here $r(I)$ is the cost of research effort $I$. Assume $r^{\prime}>0$, so a more accurate signal is more expensive. For convenience, define $v(I):=\int_{\Theta} \theta^{2} d F^{I}(\theta)$ as the second moment of $\theta^{I}$. Then condition 3 implies that $v$ is monotone increasing in I. Rewrite equation 13 as:

$$
\begin{aligned}
\mathcal{U}(\mathcal{H}) & =\max _{I \in \mathcal{I}} \max _{\mathbf{s} \in \Delta^{N}}(w(\mathbf{s}, \mathcal{H}) v(I)-r(I)) \\
& =\max _{I \in \mathcal{I}}(\bar{w}(\mathcal{H}) v(I)-r(I))
\end{aligned}
$$

Let $I^{*}(\mathcal{H})$ be the maximizer (assume it is unique, for simplicity). Then for two given hierarchies $\mathcal{H}$ and $\mathcal{H}^{\prime}$, if $\bar{w}\left(\mathcal{H}^{\prime}\right)>\bar{w}(\mathcal{H})$, standard monotone comparative statics results (Milgrom and Shannon 1994) imply that $I^{*}\left(\mathcal{H}^{\prime}\right) \geq I^{*}(\mathcal{H})$. Greater marginal social value of information $(\bar{w}(\mathbf{s}, \mathcal{H}))$ will induce higher research effort by the leader. Moreover, we have $\mathcal{U}\left(\mathcal{H}^{\prime}\right) \geq \mathcal{U}(\mathcal{H})$. As a corollary of Theorem 4.6, we can establish that the chain provides the greatest information acquisition and welfare.

Theorem 6.3 Given assumptions 1-3 and verifiability of research effort, the chain $\mathcal{C}_{N}$ induces the highest research effort and yields the greatest expected welfare in the extended game with endogenous information acquisition.

\footnotetext{
${ }^{16}$ Each $\theta^{I}$ necessarily has the same mean by the law of iterated expectations: $E\left[\theta^{I}\right]=$ $E[E[\theta \mid s]]=E[\theta], \forall I \in\left[I_{0}, I_{1}\right]$. See Rothschild and Stiglitz (1970) for the formal definition and properties of MPS.
} 
Now, we assume that we cannot write a contract contingent on the leader's research effort, either because research effort is not observable, or because it might be observable but hard to verify in court. Then the leader's research incentive comes from his private value of information, which, in general, is lower than the social value.

From Theorem 4.3, we know that the equilibrium payoff of the leader without information cost is

$$
\pi_{L}(\mathbf{s}, \mathcal{H})=\theta^{2}\left(s_{L}\left(\sum_{j \in N} k_{j}\right)-\frac{1}{2} k_{L}^{2}\right)=\frac{1}{2} \theta^{2} k_{L}(\mathbf{s}, \mathcal{H})^{2}
$$

which is monotonic in the leader's equilibrium responsive coefficient. Also, the leader's equilibrium payoff does not depend explicitly on other workers' efforts. The second equality uses the properties of $g$ and Theorem 4.3.

The optimal contract is the solution to the following program:

$$
\begin{array}{cc}
\mathcal{U}^{n}(\mathcal{H}):= & \max _{\mathbf{s} \in \Delta^{N}, I \in \mathcal{I}}(w(\mathbf{s}, \mathcal{H}) v(I)-r(I)) \\
\text { subject to: } & (\mathrm{IC}-\mathrm{L}) \quad I \in \arg \max _{I^{\prime} \in \mathcal{I}} \frac{1}{2} k_{L}(\mathbf{s}, \mathcal{H})^{2} v\left(I^{\prime}\right)-r\left(I^{\prime}\right)
\end{array}
$$

Let $\mathbf{s}^{n *}(\mathcal{H})$ and $I^{n *}(\mathcal{H})$ be the solution. IC-L is the incentive compatibility condition for the leader's research effort.

Theorem 6.4 Assume conditions 1-3 and that research effort is not verifiable. Then the chain $\mathcal{C}_{N}$ is still the most efficient hierarchy, even if we take the leader's research incentive into account. That is,

$$
\mathcal{U}^{n}\left(\mathcal{C}_{N}\right) \geq \mathcal{U}^{n}(\mathcal{H})
$$

for any simple hierarchy $\mathcal{H}$ with a single leader.

This result is quite intuitive. Since the leader's research incentive only depends on her responsive coefficient, for any $(\mathbf{s}, I)$ which satisfies IC-L under $\mathcal{H}$, we can find a new contract $\mathbf{s}^{\prime}$ for $\mathcal{C}_{N}$ such that $k_{L}\left(\mathbf{s}^{\prime}, \mathcal{C}_{N}\right)=k_{L}(\mathbf{s}, \mathcal{H})$, and $w\left(\mathbf{s}^{\prime}, \mathcal{C}_{N}\right) \geq$ $w(\mathbf{s}, \mathcal{H}){ }^{17}$ In particular, the leader's responsive coefficient is the same, so $\left(\mathbf{s}^{\prime}, I\right)$

\footnotetext{
${ }^{17}$ See proofs of Lemma 4.8 and Theorem 4.6 for the construction of $\mathbf{s}^{\prime}$.
} 
satisfies IC-L under $\mathcal{C}_{N}$. Also, $w\left(\mathbf{s}^{\prime}, \mathcal{C}_{N}\right) \geq w(\mathbf{s}, \mathcal{H})$, the marginal social value of information is also higher under the chain. Therefore, the chain both gives the leader higher incentive to acquire more accurate information and generates higher marginal social value of information. Both forces move in the same direction, so in the end, the chain wins.

Remark 4 If research effort is verifiable, then there is no conflict between choosing optimal shares and incentivizing the leader for choosing the socially optimal research effort, as one can see from equation 13. When research effort is not verifiable, choosing optimal shares and incentivizing the leader for information acquisition are in conflict.

In general, information is under-provided, i.e., $I^{n *}(\mathcal{H}) \leq I^{*}(\mathcal{H})$, because the marginal private benefit of information from the perspective of $\mathrm{L}$ is lower than the corresponding social value $\left(\pi_{L}(\mathbf{s}, \mathcal{H})<w(\mathbf{s}, \mathcal{H}) \theta^{2} \leq \bar{w}(\mathcal{H}) \theta^{2}\right)$. Of course, the maximal obtainable welfare is lower if research effort is not verifiable, i.e., $\mathcal{U}^{n}(\mathcal{H}) \leq \mathcal{U}(\mathcal{H})$. Moreover, in general $\mathbf{s}^{* n}(\mathcal{H}) \neq s^{*}(\mathcal{H})$, so we should modify the shares $s^{*}(\mathcal{H})$ to give the leader enough incentive for research. Nevertheless, the chain is the best given all these inefficiencies. Other hierarchies perform even worse.

\subsection{Applications in fund-raising}

A natural application of the model is charity fund-raising, which is similar to public good provision. Vesterlund (2003) and Andreoni (2006) emphasize the importance of leadership giving in charitable fund-raising, which serves as a signal to other givers that the charity is of high quality. Using our terminology, they show the superiority of sequential fund-raising, which corresponds to $\mathcal{L}_{(1, N-1)}$, over simultaneous fund-raising, which corresponds to $\mathcal{T}_{N}{ }^{18}$

Given the optimality of the chain structure, a charity could raise more money by implementing the chain $\mathcal{C}_{N}$, i.e., placing potential donors in a line and asking them to donate one after the other. In particular, the charity should not reveal the entire donation history to future givers. A drawback to the chain is that it

\footnotetext{
${ }^{18}$ For experimental evidence, see Protter et al., (2001,2005).
} 
requires more steps to complete the fund-raising. If delay is costly to the charity, the techniques and results of this paper might still be useful for suggesting better ways to organize the fund-raising campaign. Rather than having each donor on a separate tier, donors could be organized into subgroups, with the total donation of each subgroup revealed only to the next tier. Smaller subgroups allow for a larger number of tiers, thus raising more money, but also results in a longer delay. The optimal configuration involves a trade-off between the costs of delaying and the benefits of the funds. Carefully designed future experiments should be able to test this theory in the field.

\section{Conclusion}

This paper highlights the importance of hierarchical structures from the perspective of information flow and signaling effects associated with dissemination of information. In a team production framework, we show the optimality of the chain structure from three perspectives: maximizing dynamic signaling effects, motivating efforts of all members, and providing strong incentives for the leader's information acquisition.

This paper isolates one feature, signaling effects, of organization design. In reality, there are other forces, such as communication, adaptation and coordination, which are also relevant for the design of organizations. Also, I model leaders as the source of information. There are many other features of leadership which I have not addressed. For example, Bolton et al. (2008) show that a resolute leader can achieve a better outcome for an organization faced with conflict between adaptation and coordination as a resolute leader overestimates the precision of her prior belief and hence is less responsive to new information. They show that the coordination benefit from a resolute leader generally outweighs the cost of mal-adaptation 19 Adding these components into this model may balance the signaling effect which is dominant in this paper, thus lead to more realistic predictions about optimal hierarchies. Also, there might be other transaction costs, such as delaying, or communication costs, associated with each hierarchy. A related question is: Does the optimal hierarchy get longer and thinner, or the opposite,

\footnotetext{
${ }^{19}$ See Bolton et al. (2010) for a survey of key elements of leadership.
} 
as transaction costs drop? Adding these costs will shed some light on our understanding of real organizational design problems. A detailed analysis of these new features requires another paper, and I plan to address these issues in the future.

\section{Appendix}

\section{A Some Auxiliary Lemmas}

A few technical lemmata are presented in this section, which may be skipped on first reading. Proofs of these results are in the following sections.

The first lemma lists some properties of $g$.

Lemma A.1 Let $g(x, y)=\frac{x+\sqrt{x^{2}+4 x y}}{2}, x>0, y \geq 0$. Then:

1. (Definition) $g^{2}(x, y)=x(g(x, y)+y)$.

2. (Homogeneity) $g(\lambda x, \lambda y)=\lambda g(x, y), \forall \lambda \geq 0, x \geq 0, y \geq 0$.

3. (Monotonicity) $g(x, y)$ is strictly increasing in $x$ and $y$, and $g_{y}=\frac{x}{2 g-x} \leq 1 \leq$ $g_{x}=\frac{g+y}{2 g-x}$.

4. (Bounds) $x \leq g(x, y) \leq x+y$.

5. (Concavity) If $x>0, y>0$, then $g_{x x}<0, g_{y y}<0$ and $g_{x y}>0$.

6. (Special Values) $g(x, 0)=x$ and $g\left(\frac{y}{1+n}, n y\right)=y, \forall n, y$.

The next three lemmata are used in the proof of Theorem 4.14 .

Lemma A.2 If $x \geq 0$, then $x / 2 \leq g(x, g(x, t)+t)-g(x, t) \leq x, \quad \forall t \geq 0$. All inequalities are strict if $x>0$.

Lemma A.3 If $k, y \geq 0$, then $g(k / 3, y+g(2 k / 3, y)) \leq g(2 k / 3, y)$, with strict inequality if $y>0$ and $k>0$.

Lemma A.4 Suppose $0<k \leq 1, y \geq 0$. Define two functions $\tilde{a}, a:[0, k] \rightarrow \mathbf{R}$ as follows:

$$
\tilde{a}(e)=g(k-e, g(e, y)+y), \quad a(e)=g(e, y)
$$


Let

$$
A=\tilde{a}+a, \quad M=\tilde{a}-\frac{1}{2} \tilde{a}^{2}+a-\frac{1}{2} a^{2}
$$

Then:

1. $A(k)=A(0)<A(e), \forall e \in(0, k)$, and $M(k)=M(0)<M(e), \forall e \in(0, k)$.

2. $A$ is strictly concave in e and $A^{\prime}\left(\frac{k}{2}\right)>0$. Also, $A^{\prime}\left(\frac{2 k}{3}\right)<0$ if $y>0, A^{\prime}\left(\frac{2 k}{3}\right)=$ 0 if $y=0$; Therefore $A^{\prime}(e)>0$ if $e \in\left[0, \frac{k}{2}\right], A^{\prime}(e)<0$ if $e \in\left(\frac{2 k}{3}, k\right]$;

3. $M^{\prime}(e)>0$ on $\left[0, \frac{k}{2}\right]$, while $M^{\prime}(e)<0$ on $\left(\frac{2 k}{3}, k\right]$.

4. If $y=0$, then $A^{\prime}\left(\frac{2 k}{3}\right)=M^{\prime}\left(\frac{2 k}{3}\right)=0$, and $\frac{2 k}{3}$ is the unique maximizer of $A$ and $M$. If $y>0$, then $A^{\prime}\left(\frac{2 k}{3}\right)<0$, and $M^{\prime}\left(\frac{2 k}{3}\right)<0$.

Lemma A.5 Suppose $f:[0, \infty) \rightarrow \mathbf{R}$ is continuous and differentiable, and $f(0)=$ 0 . If $f^{\prime}(x) \leq 0$ whenever $f(x) \geq 0$, then $f(x) \leq 0, \forall x \geq 0$.

\section{A.1 Proof of Lemma A.1}

Proof Most of the calculations are straightforward.

1. If $x\left(1+\frac{y}{g}\right)=g$, then $g^{2}=x(g+y)$, or $g^{2}-x g-x y=0$, hence $g=\frac{x+\sqrt{x^{2}+4 x y}}{2}$ (drop the negative solution as $g>0$ ).

2. Trivial.

3. If $x>0, y>0$, then $g$ is differentiable in $(x, y)$. Differentiating the equation $g^{2}=x(g+y)$ with respect to $x$, we get $2 g g_{x}=g+y+x g_{x}$, hence $g_{x}=\frac{g+y}{2 g-x}>0$. Similarly, we have $2 g g_{y}=x\left(g_{y}+1\right)$, hence $g_{y}=\frac{x}{2 g-x}>0$. Note $x+y \geq g$ (part 4 below), so $g+y \geq 2 g-x$, hence $g_{x}=\frac{g+y}{2 g-x} \geq 1$. Similarly $g_{y}=\frac{x}{2 g-x} \leq 1$ since $g \geq x$.

4. This follows from $x=\frac{x+\sqrt{x^{2}+0}}{2} \leq g(x, y)=\frac{x+\sqrt{x^{2}+4 x y}}{2} \leq \frac{x+\sqrt{x^{2}+4 x y+4 y^{2}}}{2}=$ $x+y$.

5. $g(x, y)=x \zeta(y / x)$, where $\zeta(z)=\frac{1+\sqrt{1+4 z}}{2}$. It is easy to see that $\zeta^{\prime \prime}(z)<0$, hence $\zeta$ is strictly concave. So, $g_{y y}=\frac{1}{x} \zeta^{\prime \prime}(y / x)<0$ and $g_{x y}=-\frac{y}{x^{2}} \zeta^{\prime \prime}(y / x)>0$, and $g_{x x}=\frac{y^{2}}{x^{3}} \zeta^{\prime \prime}(y / x)<0$. Also $g_{x x} g_{y y}-g_{x y}^{2}=0$, therefore $g$ is concave in $(x, y)$.

6. Obviously $g(x, 0)=x$. Also, $g(1, n(n+1))=\frac{1+\sqrt{1+4 n(n+1)}}{2}=\frac{1+2 n+1}{2}=n+1$. By homogeneity of $g, g\left(\frac{y}{1+n}, n y\right)=\frac{y}{1+n} g(1, n(n+1))=y$. 


\section{A.2 Proof of Lemma A.2}

Proof If $x=0$, all terms vanish for any $t \geq 0$, hence the result holds in this case.

If $x>0$, by the Mean Value Theorem (MVT),

$$
g(x, g(x, t)+t)-g(x, t)=(g(x, t)+t-t) g_{y}(x, \zeta)
$$

for $\zeta \in(t, t+g(x, t))$. Note $g$ is concave in $y$, hence $g_{y}(x, \zeta)<g_{y}(x, t)=\frac{x}{2 g(x, t)-x}$ (Part 3, Lemma A.1). Therefore

$$
g(x, g(x, t)+t)-g(x, t)<g(x, t) g_{y}(x, t)=g(x, t) \frac{x}{2 g(x, t)-x}=x \frac{g(x, t)}{2 g(x, t)-x} .
$$

Note $g(x, t) \geq x$, hence $g(x, t) \leq 2 g(x, t)-x$, or equivalently $\frac{g(x, t)}{2 g(x, t)-x} \leq 1$. So,

$$
g(x, g(x, t)+t)-g(x, t)<x \frac{g(x, t)}{2 g(x, t)-x} \leq x \cdot 1=x
$$

For the other direction, note $g_{y}(x, \zeta)>g_{y}(x, g(x, t)+t)=\frac{x}{2 g(x, g(x, t)+t)-x}$, hence

$$
g(x, g(x, t)+t)-g(x, t)>\frac{x g(x, t)}{2 g(x, t+g(x, t))-x}
$$

Simplifying this inequality, we have 20

$$
g(x, g(x, t)+t)-g(x, t)>\frac{x}{2}>\frac{x g(x, t)}{2 g(x, t+g(x, t))-x} .
$$

Combining both directions, we get the following chain of inequalities when $x>0$ :

$$
\frac{x g(x, t)}{2 g(x, t+g(x, t))-x}<x / 2<g(x, g(x, t)+t)-g(x, t)<x \frac{g(x, t)}{2 g(x, t)-x} \leq x, \forall t \geq 0
$$

Hence Lemma A.2 is proved.

\section{A.3 Proof of Lemma A.3}

Proof If $k=0$, then both sides equal zero for any $y$, hence the result holds.

\footnotetext{
${ }^{20}(\tilde{g}-g)(2 \tilde{g}-x)>x g$, hence $2 \tilde{g}^{2}-2 g \tilde{g}-x \tilde{g}>0$, or $\tilde{g}(2 \tilde{g}-2 g-x)>0$. Thus $2 \tilde{g}-2 g-x>0$ or $\tilde{g}-g>x / 2$. Here $\tilde{g}=g(x, g(x, t)+t)$.
} 
If $k>0$, by homogeneity of $g$, we have

$$
g\left(\frac{k}{3}, g\left(\frac{2 k}{3}, y\right)+y\right)-g\left(\frac{2 k}{3}, y\right)=\frac{k}{3}\left(g\left(1, g\left(2, \frac{2 y}{k}\right)+\frac{2 y}{k}\right)-g\left(2, \frac{2 y}{k}\right)\right)
$$

So, it is sufficient to show the case for $k=3$. To this end, it is equivalent to show

$$
z(y):=g(1, g(2, y)+y)-g(2, y)<0, \forall y>0 .
$$

Note $z^{\prime}(y)=g_{y}(1, g(2, y)+y)\left(1+g_{y}(2, y)\right)-g_{y}(2, y)$. Substituting for the partial derivative of $g$ and simplifying, we have

$$
z^{\prime}(y)<0 \Longleftrightarrow 2 g(1, g(2, y)+y)-1-g(2, y)>0 .
$$

Note $2 g(1, g(2, y)+y)=g(2,2(g(2, y)+y))>g(2, g(2, y)+y)>1+g(2, y)$, $\forall y>0$, while the last inequality follows from Lemma A.2. So, $z^{\prime}(y)<0, \forall y>0$, but $z(0)=g(1,2)-g(2,0)=2-2=0$. Therefore, $z(y)<0, \forall y>0$. That finishes the proof of Lemma A.3.

\section{A.4 Proof of Lemma A.4}

Proof The proof is given in four parts.

\section{Part 1:}

Since $\tilde{a}(0)=a(k)=g(k, y), \tilde{a}(e)=a(k)=0$. Therefore $A(k)=A(0)$, and $M(0)=M(k)$.

Suppose $0<e<k$. Then 21

$$
\begin{aligned}
A(e) & =g(k-e, g(e, y)+y)+g(e, y)>g(k-e, y)+g(e, y) \\
& \geq \frac{k-e}{k} g(k, y)+\frac{e}{k} g(k, y)=g(k, y)=A(0) .
\end{aligned}
$$

For $M$, first simplify the expression using $g^{2}(x, y)=x(g(x, y)+y)$ :

$$
\begin{aligned}
M(e) & =\tilde{a}(e)-\frac{1}{2}(k-e)(\tilde{a}(e)+a(e)+y)+a(e)-\frac{1}{2} e(a(e)+y) \\
& =\left(1-\frac{k}{2}\right)(\tilde{a}(e)+a(e))+\frac{1}{2} e \tilde{a}(e)-\frac{1}{2} k y \\
& =\left(1-\frac{k}{2}\right)(\tilde{a}(e)+a(e))+\frac{1}{2} \sqrt{e^{2}(k-e)(\tilde{a}(e)+a(e)+y)}-\frac{1}{2} k y \\
& =\left(1-\frac{k}{2}\right) A(e)+\frac{1}{2} \sqrt{e^{2}(k-e)(A(e)+y)}-\frac{1}{2} k y
\end{aligned}
$$

${ }^{21}$ Note $g(e, y)$ is concave in $e$ with $g(0, y)=0$, hence $\frac{g(e, y)}{e}$ is weakly decreasing in $e \in[0, k]$. 
If $0<e<k$, then $A(e)>A(0)>0$. Also $\sqrt{e^{2}(k-e)(A(e)+y)}$ is zero if $e=0$, or $e=k$ and is positive $\forall e \in(0, k)$. Hence $M(e)>M(0)$.

\section{Part 2:}

For brevity, I use $\tilde{g}:=g(k-e, g(e, y)+y), g:=g(e, y)$. Then,

$A^{\prime}(e)=-g_{x}(k-e, g(e, y)+y)+g_{y}(k-e, g(e, y)+y) g_{x}(e, y)+g_{x}(e, y)=-\tilde{g}_{x}+\left(1+\tilde{g}_{y}\right) g_{x}$

$A^{\prime \prime}(e)=\tilde{g}_{x x}-\tilde{g}_{x y} g_{x}+\left(-\tilde{g}_{y x}+\tilde{g}_{y y} g_{x}\right) g_{x}+\left(1+\tilde{g}_{y}\right) g_{x x}$

Obviously $A^{\prime \prime}(e)<0$, because $\tilde{g}_{x x}<0, \tilde{g}_{x y}>0, \tilde{g}_{y y}<0, g_{x}>0, \tilde{g}_{y} \geq 0$, so $A$ is strictly concave. Notice that $A(0)=A(k)$, hence $A$ has a unique interior maximizer on $[0, k]$, which is given by the solution to $A^{\prime}(e)=0$. Note that

$$
A^{\prime}(e)>0 \Longleftrightarrow g_{x}>\frac{\tilde{g}_{x}}{1+\tilde{g}_{y}}
$$

From Lemma A.1. $g_{x}=\frac{g+y}{2 g-x}, \frac{\tilde{g}_{x}}{1+\tilde{g}_{y}}=\frac{\tilde{g}+g+y}{2 \tilde{g}}$. Therefore,

$$
\begin{aligned}
& g_{x}=\frac{g+y}{2 g-e}>\frac{\tilde{g}_{x}}{1+\tilde{g}_{y}}=\frac{\tilde{g}+g+y}{2 \tilde{g}} \\
& \Longleftrightarrow(g+y) 2 \tilde{g}>(2 g-e)(\tilde{g}+g+y)=\tilde{g}(2 g-e)+(g+y)(2 g-e) \\
& \Longleftrightarrow \tilde{g}(2 y+e)>(g+y)(2 g-e)=2 g^{2}+g(2 y-e)-e y \\
& \quad \quad=2 e(g+y)+g(2 y-e)-e y=g(2 y+e)+e y \\
& \Longleftrightarrow(\tilde{g}-g)(2 y+e)>e y .
\end{aligned}
$$

In the end, we have

$$
A^{\prime}(e)>(<) 0 \Longleftrightarrow \tilde{g}-g>(<) \frac{e y}{2 y+e}
$$

When $e=\frac{k}{2}, \tilde{g}-g=g(k / 2, g(k / 2, y)+y)-g(k / 2, y)>k / 4$ by Lemma A.2. Meanwhile $\frac{e y}{2 y+e}=\frac{y}{2 y+e} e<\frac{1}{2} e=k / 4$. Therefore $A^{\prime}\left(\frac{k}{2}\right)>0$. By concavity, $A^{\prime}(e)>0$ if $e \in\left[0, \frac{k}{2}\right]$.

When $e=\frac{2 k}{3}$, there are two cases. If $y=0$, then $\tilde{g}=g\left(k / 3, \frac{2 k}{3}\right)=\frac{2 k}{3}=g$. Therefore $A^{\prime}\left(\frac{2 k}{3}\right)=0$ by equation 17 . If $y>0, A^{\prime}\left(\frac{2 k}{3}\right)<0$ follows from $\tilde{g}-g=g\left(\frac{k}{3}, g\left(\frac{2 k}{3}, y\right)+y\right)-g\left(\frac{2 k}{3}, y\right)<0$, by Lemma A.3.

Therefore $A^{\prime}\left(\frac{2 k}{3}\right)<0$, if $y>0 ; A^{\prime}\left(\frac{2 k}{3}\right)=0$, if $y=0$. 


\section{Part 3:}

By equation (16),

$$
M^{\prime}(e)=\left(1-\frac{k}{2}\right) A^{\prime}(e)+\frac{1}{4}\left\{e^{2}(k-e)(A(e)+y)\right\}^{-1 / 2}\left\{e^{2}(k-e)(A(e)+y)\right\}^{\prime}
$$

where $\left\{e^{2}(k-e)(A(e)+y)\right\}^{\prime}=e(2 k-3 e)(A(e)+y)+e^{2}(k-e) A^{\prime}(e)$.

If $e \in\left[0, \frac{k}{2}\right]$, then $A^{\prime}(e)>0$ (by part 2) and $2 k-3 e>0$, so $\left\{e^{2}(k-e)(A(e)+\right.$ $y)\}^{\prime}>0$, hence $M^{\prime}(e)>0$ on $\left[0, \frac{k}{2}\right]$. Similarly, if $e \in\left(\frac{2 k}{3}, k\right]$, then $A^{\prime}(e)<0$ (by part 2) and $2 k-3 e<0$, so $\left\{e^{2}(k-e)(A(e)+y)\right\}^{\prime}<0$. Thus $M^{\prime}(e)<0$ on $\left(\frac{2 k}{3}, k\right]$.

\section{Part 4:}

If $y=0$, at $e=\frac{2 k}{3}, A^{\prime}\left(\frac{2 k}{3}\right)=0$, so $\left.\left\{e^{2}(k-e)(A(e)+y)\right\}^{\prime}\right|_{e=\frac{2 k}{3}}=0$ (both $A^{\prime}(e)$ and $2 k-3 e$ vanish at this point), therefore $M^{\prime}\left(\frac{2 k}{3}\right)=0$. Also, in this case, if $e<\frac{2 k}{3}$, $A^{\prime}(e)>0$, hence $M^{\prime}(e)>0$. If $e>\frac{2 k}{3}$, then $A^{\prime}(e)<0$, hence $M^{\prime}(e)<0$. Thus $\frac{2 k}{3}$ is the unique maximizer of both $M$ and $A$ on $[0, k]$.

If $y>0$, then at $e=\frac{2 k}{3}, A^{\prime}\left(\frac{2 k}{3}\right)<0$. Therefore $\left.\left\{e^{2}(k-e)(A(e)+y)\right\}^{\prime}\right|_{e=\frac{2 k}{3}}<0$ (note $2 k-3 e$ vanishes at this point), hence $M^{\prime}\left(\frac{2 k}{3}\right)<0$. Moreover, $M$ is decreasing on $\left(\frac{2 k}{3}, k\right]$ by part 3 , so the maximizer of $M$ is less than $\frac{2 k}{3}$.

\section{A.5 Proof of Lemma A.5}

Proof For $\epsilon>0$, let $g(x)=f(x)-\epsilon(1+x)$. If $g(x) \geq 0$, then $f(x) \geq 0$, and $f^{\prime}(x) \leq 0$. Hence $g^{\prime}(x)=f^{\prime}(x)-\epsilon<0$. We claim that $g(x) \leq 0$ for all $x \geq 0$. Suppose $g(\bar{x})>0$. Then let $\hat{x}=\inf \{x \geq 0 \mid g(x) \geq 0\}$. Note $g(0)<0$, hence $\hat{x} \neq 0$. Moreover $g(\hat{x})=0$ and $g(x)<0$ for $\forall x<\hat{x}$. Therefore $g^{\prime}(\hat{x}) \geq 0$. Also, $g(\hat{x})=0$, so $g^{\prime}(\hat{x})<0$, hence we get a contradiction. Therefore $g(x) \leq 0, \forall x$. This implies $f(x) \leq \epsilon(1+x)$. This holds for any positive $\epsilon$. Taking the limit as $\epsilon$ goes to zero, we have $f(x) \leq 0, \forall x$.

\section{B Omitted Proofs}

All omitted proofs are given in this section.

\section{B.1 Proof of Theorem 4.1}

Before the proof, we need an auxiliary lemma. 
Lemma B.1 For a simple hierarchy $\mathcal{H}$, the following are true:

A. $\left\{D F^{j}: j \in N^{k}\right\}$ is a partition of $N^{k+1}$, for $k=1,2, \cdots, h-1$; that is, $\cup_{j \in N^{k}} D F^{j}=N^{1+k}$ and for $j \neq i, D F^{j} \cap D F^{i}=\emptyset$.

B. If $i, j \in N^{k}$ and $i \neq j$, then $F^{i} \cap F^{j}=\emptyset$.

C. For any two members $i, j$, there is at most one path from $i$ to $j$.

Proof For A, the union of $D F^{j}$ is $N^{k+1}$ by part (b) of Definition 3 . The disjointness of these sets follows from simplicity of the hierarchy. B and $\mathrm{C}$ follow from $\mathrm{A}$ using induction.

Proof of Theorem 4.1 If $\mathcal{H}$ is simple, then every worker except the leaders has a unique predecessor, hence a unique source of information. Also, $F^{i}$ identifies the set of players whose beliefs can be influenced by $i$ 's effort. Lemma B.1 shows that if $i, j \in N^{k}$, then $F^{i} \cap F^{j}=\emptyset$, i.e, $i$ and $j$ have no common followers, which makes the equilibrium characterization much easier.

We are interested in separating equilibrium, in which any player's effort reveals his belief about the state. For each player $i \in \mathcal{N}$, let $\tilde{x}_{i}: \Theta \rightarrow[0,+\infty)$ denote player $i$ 's optimal effort given his belief about the state. The equilibrium condition is that for any $i$,

$$
\left.\tilde{x}_{i}(\theta) \in \arg \max _{x_{i} \in \mathbf{R}^{+}} s_{i} \theta\left(x_{i}+\sum_{j \in F^{i}} \tilde{x}_{j}\left(\tilde{x}_{i}^{-1}\left(x_{i}\right)\right)\right)\right)-c\left(x_{i}\right) .
$$

The first-order condition for equation 18 is

$$
s_{i} \theta\left(1+\sum_{j \in F^{i}} \frac{\tilde{x}_{j}^{\prime}\left(\tilde{x}_{i}^{-1}\left(x_{i}\right)\right)}{\tilde{x}_{i}^{\prime}\left(\tilde{x}_{i}^{-1}\left(x_{i}\right)\right)}\right)-c^{\prime}\left(x_{i}\right)=0, \text { when } x_{i}=\tilde{x}_{i}(\theta) .
$$

Note $\tilde{x}_{i}^{-1}\left(x_{i}\right)=\theta$ if $x_{i}=\tilde{x}_{i}(\theta)$. Simplifying the above expression, we get:

$$
s_{i} \theta\left(1+\sum_{j \in F^{i}} \frac{\tilde{x}_{j}^{\prime}(\theta)}{\tilde{x}_{i}^{\prime}(\theta)}\right)=c^{\prime}\left(\tilde{x}_{i}(\theta)\right) .
$$

This must hold for any $\theta \in \Theta$, which is just equation 2 . 
Remark 5 In equation 18, we only consider the contributions of players in $F^{i}$ and $i$, but ignore the contributions of others workers. The reason is other workers cannot be influenced by i's effort, hence their contributions only affect i's equilibrium payoff and do not affect $i$ 's incentive for signaling. By Lemma B.1, we can isolate player i's problem from other players on the same level because they have disjoint sets of followers.

Remark 6 In general, we need to specify initial conditions to solve for ordinary differential equations. We do not need to do so here because $\tilde{x}_{i}(0)=0, \forall i \in \mathcal{N}$ is implicitly implied by equation 2 by setting $\theta=0$. If $\min \Theta=\underline{\theta}>0$, then the initial condition for equation 2 (see Mailath, 1987) is fixed by requiring that the "worst" type, $\underline{\theta}$, get his maximal utility given that he is identified as the worst type; in other words, $\tilde{x}_{i}(\underline{\theta})=c^{-1}\left(s_{i} \underline{\theta}\right)$. In general, no explicit solutions exist when $\underline{\theta}>0$ even with quadratic disutility function.

\section{B.2 Proof of Lemma 4.8:}

Part 1 Let $\rho$ be the inverse map of $\sigma$. Then $\rho$ is also a permutation of $\mathcal{N}$. It suffices to show:

$$
(+) \quad k_{\rho(i)}\left(\mathbf{s}, \mathcal{C}_{(\mathcal{N})}^{\sigma}\right) \geq k_{\rho(i)}(\mathbf{s}, \mathcal{H}), \forall i \in \mathcal{N}
$$

We prove $(+)$ by induction on $i$ from bigger $i$ to smaller $i$.

For $i=N$, we know that $\rho(N)$ is the worker on the last level, hence has no followers in the chain. So $\sigma(\rho(N))=N \geq \sigma(i), \forall i$. Therefore $\rho(N)$ has no follower under $\mathcal{H}$. Thus, $k_{\rho(i)}\left(\mathbf{s}, \mathcal{C}_{(\mathcal{N})}^{\sigma}\right)=s_{\rho(N)}=k_{\rho(i)}(\mathbf{s}, \mathcal{H})$.

Suppose $(+)$ holds for all $i$ greater than or equal to $K$. If $i=K-1$, then by Theorem 4.3

$$
k_{\rho(K-1)}(\mathbf{s}, \mathcal{H})=g\left(s_{\rho(K-1)}, \sum_{j \in F^{\rho(K-1)}} k_{j}(\mathbf{s}, \mathcal{H})\right)
$$

By monotonicity of $\sigma$, we have

$$
F^{\rho(K-1)} \subset\{j \mid \sigma(j)>\sigma(\rho(K-1)=K-1\}=\{j \mid \sigma(j) \geq K\}=\{\rho(l) \mid l \geq K\} .
$$


Therefore,

$$
\begin{aligned}
k_{\rho(K-1)}(\mathbf{s}, \mathcal{H}) & =g\left(s_{\rho(K-1)}, \sum_{j \in F^{\rho(K-1)}} k_{j}(\mathbf{s}, \mathcal{H})\right) \\
& \leq g\left(s_{\rho(K-1)}, \sum_{l \geq K} k_{\rho(l)}(\mathbf{s}, \mathcal{H})\right) \\
& \leq g\left(s_{\rho(K-1)}, \sum_{l \geq K} k_{\rho(l)}\left(\mathbf{s}, \mathcal{C}_{(\mathcal{N})}^{\sigma}\right)\right) \quad \text { (by induction) } \\
& =k_{\rho(K-1)}\left(\mathbf{s}, \mathcal{C}_{(\mathcal{N})}^{\sigma}\right)
\end{aligned}
$$

Therefore, $(+)$ holds for $K-1$. By induction, $(+)$ holds for any $i=1, \cdots, N$.

Part 2 We construct $\tilde{\mathbf{s}}$ step-by-step to satisfy the following conditions:

$$
(++) \quad k_{\rho(i)}\left(\tilde{\mathbf{s}}, \mathcal{C}_{(\mathcal{N})}^{\sigma}\right)=k_{\rho(i)}(\mathbf{s}, \mathcal{H}), \forall i \in \mathcal{N}
$$

For $i=N$, let $\tilde{s}_{\rho(N)}=s_{\rho(N)}$. Note $\rho(N)$ has no followers under $\mathcal{H}$ or $\mathcal{C}_{(\mathcal{N})}^{\sigma}$, so in this case, $k_{\rho(N)}\left(\tilde{\mathbf{s}}, \mathcal{C}_{(\mathcal{N})}^{\sigma}\right)=\tilde{s}_{\rho(N)}=s_{\rho(N)}=k_{\rho(i)}(\mathbf{s}, \mathcal{H})$. So $(++)$ holds for $i=N$.

Suppose we have constructed $\tilde{s}_{i}$ for all $i \geq K$. Define $\epsilon \geq 0$ such that:

$$
g\left(s_{\rho(K-1)}, \sum_{j \in F^{\rho(K-1)}} k_{j}(\mathbf{s}, \mathcal{H})\right)=g\left(s_{\rho(K-1)}-\epsilon, \sum_{l \geq K} k_{\rho(l)}(\mathbf{s}, \mathcal{H})\right) .
$$

This $\epsilon$ always exists by continuity of $g$, because the right hand side is bigger than the left hand side if $\epsilon=0$, and the right hand side is zero if $\epsilon=s_{\rho(K-1)}$. Let $\tilde{s}_{\rho(K-1)}=s_{\rho(K-1)}-\epsilon \leq s_{\rho(K-1)}$. Then

$$
\begin{aligned}
k_{\rho(K-1)}(\mathbf{s}, \mathcal{H}) & =g\left(s_{\rho(K-1)}, \sum_{j \in F^{\rho(K-1)}} k_{j}(\mathbf{s}, \mathcal{H})\right) \\
& =g\left(s_{\rho(K-1)}-\epsilon, \sum_{l \geq K} k_{\rho(l)}(\mathbf{s}, \mathcal{H})\right) \\
& =g\left(\tilde{s}_{\rho(K-1)}, \sum_{l \geq K} k_{\rho(l)}\left(\tilde{\mathbf{s}}, \mathcal{C}_{(\mathcal{N})}^{\sigma}\right)\right) \quad \text { by induction } \\
& =k_{\rho(K-1)}\left(\tilde{\mathbf{s}}^{\left.\mathcal{C}_{(\mathcal{N})}^{\sigma}\right)}\right.
\end{aligned}
$$

Therefore, $(++)$ holds for $i=K-1$. The results follow by induction. 


\section{B.3 Proof of Theorem 4.9}

Proof Suppose $\mathbf{s}=\left\{s_{i}, i \in \mathcal{N}\right\}$ is optimal for $\phi\left(t_{2}, \mathcal{H}\right.$ ) (an optimum always exists by continuity of $w(\mathbf{s}, \mathcal{H})$ and compactness of $\left.\Delta^{N}\right)$. Note $\sum_{i \in \mathcal{N}} s_{i}=t_{1}<t_{2}$ and let $\Delta=t_{2}-t_{1}>0$. Choose one terminal worker, say $b$. Suppose his share is $s_{b} \in\left[0, t_{1}\right]$. Obviously, $b$ has no followers. Let $P^{b}$ be the set of $b$ 's predecessors. Also, we have to remove the workers with zero shares, so define $M=\left\{j \in P^{b} \mid s_{j}>0\right\}$. It is easy to see that $k_{j}(\mathbf{s}, \mathcal{H})$ is strictly increasing in $s_{b}$ if $j \in M$.

Let $\hat{e}=\Delta+\sum_{j \in M} s_{j}>0$. We claim that:

Claim B.2 There exist functions $\left\{f_{j}, j \in M\right\}$ defined on $e \in[0, \hat{e}]$ such that:

(1) $\forall j \in M, f_{j}$ is continuous and nonnegative in e with $f_{j}(0)=0$.

(2) $s_{j}-f_{j}(e)>0$.

(3) $k_{j}(\tilde{\mathbf{s}}(e))=k_{j}(\mathbf{s}), j \neq b$, and $k_{b}(\tilde{\mathbf{s}}(e))=s_{b}+e$, where $\tilde{\mathbf{s}}(e)$ is the shares derived from $\mathbf{s}$ by adjusting $s_{b} \rightarrow s_{b}+e$ and $s_{j} \rightarrow s_{j}-f_{j}(e), j \in M$, and keeping all other workers' shares fixed.

If this claim is true, then define $\eta(e)=\Delta-e+\sum_{j \in M} f_{j}(e), e \in[0, \hat{e}]$. Notice that the summation of shares for $\tilde{\mathbf{s}}(e)$ is

$$
|\tilde{\mathbf{s}}(e)|=\sum_{i \in \mathcal{N}} s_{j}+e-\sum_{j \in M} f_{j}(e)=t_{1}+e-\sum_{j \in M} f_{j}(e)=t_{1}+\Delta-\eta(e)=t_{2}-\eta(e),
$$

which varies with $e$. Also, $\eta(0)=\Delta-0+0>0$, and

$$
\eta(e)=\Delta-e+\sum_{j \in M} f_{j}(e) \leq \Delta-e+\sum_{j \in M} s_{j} \quad\left(\text { note } f_{j}(e)<s_{j}\right)
$$

Therefore $\eta(e)<0$ if $e>\Delta+\sum_{j \in M} s_{j}=\hat{e}$. By the Mean Value Theorem, there exists $e$ such that $\eta(e)=0$. Let $\bar{e}=\min \{e \geq 0 \mid \eta(e)=0\}$; this number exists and is finite.

For $e \in[0, \bar{e}), \eta(e)>0$, hence $|\tilde{\mathbf{s}}(e)|=t_{2}-\eta(e) \leq t_{2} \leq 1$. The responsive coefficients of all workers except $b$ are the same under $\tilde{\mathbf{s}}(e)$ by part 3 Claim B.2. and $k_{b}(\tilde{\mathbf{s}}(e))=s_{b}+e$ (b has no followers), which is increasing in $e$.

$$
\frac{\partial w(\tilde{\mathbf{s}}(e), \mathcal{H})}{\partial e}=\frac{\partial}{\partial e}\left\{s_{b}+e-\frac{1}{2}\left(s_{b}+e\right)^{2}\right\}=1-\left(s_{b}+e\right)>1-|\tilde{\mathbf{s}}(e)| \geq 1-t_{2} \geq 0
$$


Therefore, the aggregate welfare $w(\tilde{\mathbf{s}}(e), \mathcal{H})$ is strictly increasing as we increase $e \in[0, \bar{e}]$, while for $e=0, \tilde{s}(0)=\mathbf{s}$, and for $e=\bar{e},|\tilde{\mathbf{s}}(\bar{e})|=t_{2}-\eta(\bar{e})=t_{2}-0=t_{2}$. In the end, we have:

$$
\begin{aligned}
\phi\left(t_{1}, \mathcal{H}\right) & =w(\mathbf{s}, \mathcal{H})=w(\tilde{\mathbf{s}}(0), \mathcal{H}) \\
& <w(\tilde{\mathbf{s}}(\bar{e}), \mathcal{H}) \leq \max _{\mathbf{s} \geq 0, \sum s_{j}=t_{2}} w(\mathbf{s}, \mathcal{H})=\phi\left(t_{2}, \mathcal{H}\right)
\end{aligned}
$$

which completes the proof of Theorem 4.9.

Proof of Claim B.2 We can construct these functions step-by-step. For each $e \geq 0, j \in M$, define $f_{j}(e)$ as the unique solution to the following

$$
g\left(s_{j}, e+\sum_{l \in F^{j}} k_{l}\right)=g\left(s_{j}-f_{j}(e), \sum_{l \in F^{j}} k_{l}\right)
$$

The solution $f_{j}(e)$ exists and is unique by continuity of $g$ and the fact that $j \in M$ and $s_{j}>0$. Also, $f_{j}$ is continuous by the implicit function theorem.

Last, we need to check the three conditions in Claim B.2, Parts 1 and 2 are obviously true by construction. For part 3, we prove this by induction on the level of members.

If $j \in N^{h}$, then $k_{j}(\tilde{\mathbf{s}}(e))=\tilde{s}_{j}(e)=s_{j}$ if $j \neq b$, and $k_{b}(\tilde{\mathbf{s}}(e))=\tilde{s}_{b}(e)=s_{b}+e$. Suppose part 3 holds for any member on levels higher than $K$. Suppose $j \in N^{K-1}$. There are two cases.

(1) Suppose $j \in M$. Hence $j \in P^{b}$, and $b \in F^{j}$. Then

$$
\begin{aligned}
k_{j}(\tilde{\mathbf{s}}(e)) & =g\left(s_{j}-f_{j}(e), \sum_{l \in F^{j}} k_{l}(\tilde{\mathbf{s}}(e))\right) \\
& =g\left(s_{j}-f_{j}(e), k_{b}(\tilde{\mathbf{s}}(e))+\sum_{l \in F^{j}, l \neq b} k_{l}(\tilde{\mathbf{s}}(e))\right) \\
& =g\left(s_{j}-f_{j}(e), s_{b}+e+\sum_{l \in F^{j}, l \neq b} k_{l}(\mathbf{s})\right) \quad(\text { by induction }) \\
& \left.=g\left(s_{j}-f_{j}(e), e+\sum_{l \in F^{j}} k_{l}(\mathbf{s})\right)=g\left(s_{j}, e+\sum_{l \in F^{j}} k_{l}\right) \quad \text { (by definition of } f_{j}(e)\right) \\
& =k_{j}(\mathbf{s})
\end{aligned}
$$

The fourth equality follows by induction, since the set $F^{j}$ must lie on a higher level than $j$. 
(2) If $j \notin M$, then $b \notin F^{j}$. Then

$$
k_{j}(\tilde{\mathbf{s}}(e))=g\left(s_{j}, \sum_{l \in F^{j}} k_{l}(\tilde{\mathbf{s}}(e))\right)=g\left(s_{j}, \sum_{l \in F^{j}} k_{l}(\mathbf{s})\right)=k_{j}(\mathbf{s})
$$

By induction, part 3 holds for any member $j$.

This example shows that the result is not as obvious as it appears.

Example 5 There exists a hierarchy $\mathcal{H}$ and two shares $\mathbf{s}, \mathbf{s}^{\prime}$ with $\mathbf{s}^{\prime} \geq \mathbf{s}$, but $w(\mathbf{s}, \mathcal{H})>w\left(\mathbf{s}^{\prime}, \mathcal{H}\right)$.

For the chain $A \rightarrow B \rightarrow C$ with $s_{A}=0.8, s_{B}=s_{C}=0.1$, we have $k_{A}\left(\mathbf{s}, C_{3}\right)=$ $1.00782>1$. Reduce the share of $s_{A}$ by $1 \%$, which will reduce $k_{A}$ to 0.99737 without affecting $k_{B}, k_{C}$. Note $0.99737-\frac{1}{2} 0.99737^{2}>1.00782-\frac{1}{2} 1.00782^{2}$. The new shares add up to only $99 \%$, but yield higher aggregate welfare. The problem is that the shares are not optimally adjusted as we did in Theorem 4.9 .

\section{B.4 Proof of Lemma 4.11}

Proof For the chain, the responsive coefficients $k_{i}$ and $k_{i+1}$ are related by the following:

$$
k_{i}-k_{i+1}=g(x, g(x, t)+t)-g(x, t)
$$

where $x=\frac{1}{N}>0, t=\sum_{j>i+1} k_{j} \geq 0$. By Lemma A.2, $k_{i}-k_{i+1}$ lies between $x / 2$ and $x$, in other words, $\frac{1}{2 N}<k_{i}-k_{i+1}<\frac{1}{N}, i=1, \cdots, N-1$. Taking summations, we have:

$$
\frac{N+1-i}{2 N}<k_{i}<\frac{N+1-i}{N} \quad i=1, \cdots, N-1
$$

which completes the proof of Lemma 4.11 .

\section{B.5 Proof of Proposition 4.12}

Proof Based on the estimates of $k_{i}$ from Lemma 4.11, we have:

$$
\begin{aligned}
\sum_{k=1}^{N}\left(\frac{N+1-i}{2 N}-\frac{1}{2}\left(\frac{N+1-i}{2 N}\right)^{2}\right) & \leq w\left(\mathbf{s}^{e q}, \mathcal{C}_{N}\right)=\sum_{k=1}^{N}\left(k_{i}-\frac{1}{2} k_{i}^{2}\right) \\
& \leq \sum_{k=1}^{N}\left(\frac{N+1-i}{N}-\frac{1}{2}\left(\frac{N+1-i}{N}\right)^{2}\right)
\end{aligned}
$$


Simplifying the terms, we get

$$
\frac{(1+N)(-1+10 N)}{48 N} \leq w\left(\mathbf{s}^{e q}, \mathcal{C}_{N}\right) \leq \frac{(1+N)(-1+4 N)}{12 N}
$$

Therefore

$$
\frac{5}{24} N+\frac{1}{6} \leq w\left(\mathbf{s}^{e q}, \mathcal{C}_{N}\right) \leq \frac{1}{3} N+\frac{1}{4}
$$

For a large team with equal shares, $w\left(\mathbf{s}^{e q}, \mathcal{C}_{N}\right)$ grows at least linearly in $N$.

\section{B.6 Proof of Proposition 4.13}

Proof This proof is quite similar to the proof of Lemma 4.11 .

For the chain structure, the the responsive coefficients $k_{i}$ and $k_{i+1}$ are related by the following:

$$
\begin{aligned}
k_{i}-k_{i+1} & =g(x, g(y, t)+t)-g(y, t) \\
& =\underbrace{(g(x, g(y, t)+t)-g(y, g(y, t)+t))}_{I}+\underbrace{(g(y, g(y, t)+t)-g(y, t))}_{I I}
\end{aligned}
$$

where $x=s_{i}, y=s_{i+1}, t=\sum_{j>i+1} k_{j}$. Note that $x \leq y$ and $t \geq 0$ by assumption. The first term $I=g(x, g(y, t)+t)-g(y, g(y, t)+t)=(x-y) g_{x}(\zeta, g(y, t)+t)$ for some $\zeta$ by the Mean Value Theorem. Note $g_{x} \geq 1$ and $x-y \leq 0$, so $I \leq$ $(x-y) g_{x}(x, \zeta) \leq(x-y)$. The second term $I I=g(y, g(y, t)+t)-g(y, t) \leq y$ by Lemma A.2. Therefore, $k_{i}-k_{i+1}=I+I I \leq(x-y)+y=x=s_{i}$. Taking summations, we have

$$
k_{i} \leq \sum_{j \geq i}\left(k_{j}-k_{j+1}\right) \leq \sum_{j \geq i} s_{j}
$$

Note the sum of all shares is one, so $k_{i} \leq 1$.

If all the shares $s_{i}$ are positive, then the second term $I I<y=s_{i+1}$, which implies $k_{i}-k_{i+1}<s_{i}$, therefore $k_{i}<1$.

\section{B.7 Proof of Theorem 4.14}

Proof The proof consists of two steps.

Step 1: Proof that $\frac{s_{i}^{*}}{s_{i+1}^{*}} \in[0.5,1)$ 
Suppose $\mathbf{s}^{*}$ is optimal for the chain. We want to prove that $\frac{s_{i}^{*}}{s_{i+1}^{*}} \in[0.5,1)$ for any $i$. Let $k=s_{i}^{*}+s_{i+1}^{*}, e=s_{i+1}^{*}$. Then it is easy to see that

$$
\frac{s_{i}^{*}}{s_{i+1}^{*}} \in[0.5,1) \Longleftrightarrow \frac{e}{k}=\frac{1}{1+\frac{s_{i}^{*}}{s_{i+1}^{*}}} \in\left(\frac{1}{2}, \frac{2}{3}\right]
$$

We prove this is true by contradiction.

Suppose $\frac{e}{k} \in\left[0, \frac{1}{2}\right]$, or $e \in\left[0, \frac{k}{2}\right]$. Then from Lemma A.4 we have $A^{\prime}(e)>$ $0, M^{\prime}(e)>0$. So for small $\delta>0$, the following two conditions hold:

$$
A(e+\delta)>A(e) \text { and } M(e+\delta)>M(e)
$$

Hence we can define the new shares $\hat{\mathbf{s}}$ by moving $\delta$ from $i$ to $i+1$ in $\mathbf{s}^{*}$. Obviously, this will not change the incentives of workers after $i+1$. Also by equation (19), the new shares $\hat{\mathbf{s}}$ satisfy:

$$
\begin{aligned}
& \hat{k}_{l}=k_{l}, l>i+1 \\
& \hat{k}_{i}+\hat{k}_{i+1}>k_{i}+k_{i+1} \\
& \hat{k}_{i}-\frac{1}{2}\left(\hat{k}_{i}\right)^{2}+\hat{k}_{i+1}-\frac{1}{2}\left(\hat{k}_{i+1}\right)^{2}>k_{i}-\frac{1}{2}\left(k_{i}\right)^{2}+k_{i+1}-\frac{1}{2}\left(k_{i+1}\right)^{2}
\end{aligned}
$$

By induction, we also have $\hat{k}_{l}>k_{l}, l<i$.

Because we do not know the range of $\hat{k}_{l}$, we cannot argue that $w\left(\hat{\mathbf{s}}, \mathcal{C}_{N}\right)>$ $w\left(\mathbf{s}^{*}, \mathcal{C}_{N}\right)$. Instead, we apply the same trick as before: we reduce the share of $i-1$ by a suitable amount $\epsilon_{i-1} \geq 0$ such that

$$
g\left(s_{i-1}-\epsilon_{i-1}, \hat{k}_{i}+\hat{k}_{i+1}+\sum_{j>i+1} \hat{k}_{j}\right)=g\left(s_{i-1}, k_{i}+k_{i+1}+\sum_{j>i+1} k_{j}\right)
$$

This is always feasible by continuity of $g$ and equation (21). Then do the same operations for player $i-2, i-3$ through player 1 such that their responsive coefficients for the reduced shares are the same as those with $\mathbf{s}^{*}$. Call $\tilde{\mathbf{s}}$ the reduced share profile. Then $|\tilde{\mathbf{s}}|=\beta<1$ by construction. Therefore, $w\left(\tilde{\mathbf{s}}, \mathcal{C}_{N}\right)>w\left(\mathbf{s}^{*}, \mathcal{C}_{N}\right)$ by equation 22 . On the other hand,

$$
w\left(\tilde{\mathbf{s}}, \mathcal{C}_{N}\right) \leq \phi\left(\beta, \mathcal{C}_{N}\right) \leq \phi\left(1, \mathcal{C}_{N}\right)=w\left(\mathbf{s}^{*}, \mathcal{C}_{N}\right)
$$

So we get a contradiction. Hence it is impossible to have $e \in\left[0, \frac{k}{2}\right]$. 
Similarly, it is impossible to have $\frac{e}{k} \in\left(\frac{2}{3}, 1\right]$ by using Lemma A.4 to get a contradiction.

For the last two workers, we can even show that $s_{N-1}=\frac{1}{2} s_{N}$. If this is not the case, then we can move part of the share from one worker to the other such that the ratio is $1 / 2$. Notice that in this case we will have $A(2 k / 3)>A(e)$, and $M(2 k / 3)>M(e)$ (Part 4 of Lemma A.4). We get a contradiction by using the same method as above.

Step 2: Proof that $k_{1}>k_{2}>\cdots>k_{N-1}=k_{N}$

Notice that $s_{N-1}^{*}=0.5 s_{N}^{*}$, so $k_{N-1}=g\left(0.5 s_{N}^{*}, s_{N}^{*}\right)=s_{N}^{*}=k_{N}$.

For any other pair of players $\left\{s_{i}, s_{i+1}\right\}, i \neq N-1$, let $k=s_{i}+s_{i+1}, \bar{e}=s_{i+1}$. If $k_{i} \leq k_{i+1}$, then $\tilde{g}-g=k_{i+1}-k_{i} \leq 0$, and $\frac{\bar{e} y}{2 y+\bar{e}}>0(y>0$ in this case because $i+1$ is not the terminal worker). Therefore

$$
A^{\prime}(\bar{e})<0, \text { or } \tilde{a}^{\prime}(\bar{e})+a^{\prime}(\bar{e})<0
$$

by equation 17. Also, by monotonicity of $s_{i}$ in Step 1 and Proposition 4.13 we have $k_{i} \leq k_{i+1}<1$, therefore $1>a(\bar{e}) \geq \tilde{a}(\bar{e})$. Then

$M^{\prime}(e)=(1-\tilde{a}(e)) \tilde{a}^{\prime}(e)+(1-a(e)) a^{\prime}(e)=(1-\tilde{a}(e))\left(\tilde{a}^{\prime}(e)+a^{\prime}(e)\right)+(\tilde{a}(e)-a(e)) a^{\prime}(e)$

which is strictly negative at $e=\bar{e}$, since $(1-\tilde{a}(\bar{e}))\left(\tilde{a}^{\prime}(\bar{e})+a^{\prime}(\bar{e})\right)<0$, and $(\tilde{a}(\bar{e})-$ $a(\bar{e})) a^{\prime}(\bar{e}) \leq 0$. Therefore we have shown that

$$
A^{\prime}(\bar{e})<0 \text {, and } M^{\prime}(\bar{e})<0
$$

This means that we can reduce $s_{i+1}$ by a small amout $\delta>0$, and increase $s_{i}$ by the same amount $\delta$, such that:

$$
A(\bar{e}-\delta)>A(\bar{e}), \text { and } M(\bar{e}-\delta)>M(\bar{e})
$$

The same procedure can be used to get a contradiction. So $k_{i}>k_{i+1}$ for $\forall i \neq N-1$. Hence, the proof is complete.

\section{B.8 Proof of Theorem 5.1}

Proof We want to show that the linear functions given by Theorem 5.1 are part of a separating equilibrium with the pessimistic belief assumption. For brevity, let 
$k_{i}=k_{i}(\mathbf{s}, \mathcal{H})$. Now fix a player $i \in N^{k}$. If $k=h$, i.e., $i$ is a terminal worker, then obviously $k_{i}=s_{i}$. Now suppose $k<h$. Let $F^{i}$ be the set of followers of $i$. There are two possible deviations for $i$, upward and downward.

If $i$ deviates downward (we only consider one player deviating, so all other workers on level $k$ are "telling the truth"), then all the players in $F^{i}$ will use $i$ 's effort to update beliefs by the pessimistic belief assumption and choose efforts accordingly. No profitable downward deviating means that:

$$
k_{i} \theta \in \arg \max _{x \leq k_{i} \theta} s_{i} \theta\left(x+\sum_{j \in F^{i}} k_{j}\left(\frac{x}{k_{i}}\right)\right)-\frac{1}{2} x^{2}
$$

Using the first order condition, this is equivalent to

$$
s_{i} \theta\left(1+\sum_{j \in F^{i}} \frac{k_{j}}{k_{i}}\right) \geq k_{i} \theta \Longleftrightarrow k_{i} \geq g\left(s_{i}, \sum_{j \in F^{i}} k_{j}\right)
$$

Clearly $k_{i}=g\left(s_{i}, \sum_{j \in F^{i}} k_{j}\right)$ satisfies the condition above (this is actually an equality in this case).

If $i$ deviates upward, the situation is a little bit different, as not all the followers will "listen to" $i$ 's effort. Let

$$
F_{c}^{i}=\left\{j \in F^{i} \mid \text { there is no path from } l \text { to } j \text { for } l \in N^{k}, l \neq i\right\} .
$$

These workers will follow $i$ because they cannot detect $i$ 's upward deviation as $i$ is the only source of information for them. The workers in $F^{i} \backslash F_{c}^{i}$ will not be affected by $i$ 's upward deviation as they get at least one other signal saying that the state is $\theta$. Thus, no profitable upward deviation means that:

$$
k_{i} \theta \in \arg \max _{x \geq k_{i} \theta} s_{i} \theta\left(x+\sum_{j \in F_{c}^{i}} k_{j}\left(\frac{x}{k_{i}}\right)\right)-\frac{1}{2} x^{2}
$$

Using the first order condition, this is equivalent to

$$
s_{i} \theta\left(1+\sum_{j \in F_{c}^{i}} \frac{k_{j}}{k_{i}}\right) \leq k_{i} \theta \Longleftrightarrow k_{i} \geq g\left(s_{i}, \sum_{j \in F_{c}^{i}} k_{j}\right)
$$

Obviously, $k_{i}=g\left(s_{i}, \sum_{j \in F^{i}} k_{j}\right) \geq g\left(s_{i}, \sum_{j \in F_{c}^{i}} k_{j}\right)$ satisfies this condition, as $F_{c}^{i} \subset$ $F^{i}$. 
Combing these two results, we have shown that $k_{i} \theta$ is $i$ 's best response given all the other players' best responses, thus we have verified that it is part of a separating equilibrium supported by pessimistic beliefs.

Remark 7 As we have seen implicitly in the above proof, any number in the interval $\left[g\left(s_{i}, \sum_{j \in F_{c}^{i}} k_{j}\right), g\left(s_{i}, \sum_{j \in F^{i}} k_{j}\right)\right]$ is a possible choice for $i$ 's equilibrium responsive coefficient. We choose the largest one in that interval. The same thing happens in the $V$ structure (Section 2). I conjecture that the equilibrium efforts characterized in Theorem 5.1 are the upper bounds of equilibrium efforts among all separating equilibria supported by pessimistic beliefs as I show for the $V$ structure in section 2 .

\section{B.9 Proof of Theorem 5.3}

Proof Suppose $\mathbf{s}$ is optimal for $\phi(t, \mathcal{H})$, i.e., $\phi(t, \mathcal{H})=w(\mathbf{s}, \mathcal{H})$. We claim that there exists a contract $\mathbf{s}^{\prime}$ such that $w(\mathbf{s}, \mathcal{H})=w\left(\mathbf{s}^{\prime}, \mathcal{H}+i j\right)$ and $\mathbf{s}^{\prime} \leq \mathbf{s}$. Then the proposition follows directly from this claim and Theorem 4.9 .

To show the claim, first we claim that there exists a $\delta \geq 0$ such that:

$$
g\left(s_{i}, \sum_{t \in F^{i}} k_{t}(\mathbf{s}, \mathcal{H})\right)=g\left(s_{i}-\delta, \sum_{t \in F_{a}^{i}} k_{t}(\mathbf{s}, \mathcal{H})\right)
$$

Here $F_{a}^{i}$ is the set of $i$ 's followers in $\mathcal{H}+i j$, which is larger than $F^{i}$ because a link from $i$ to $j$ was added. The existence of $\delta$ follows from continuity of $g$. By induction, we can keep weakly reducing all players on the top of $l$ while keeping their incentives the same under the two hierarchies. As everyone has weakly more followers after adding the link, we can always do that. In the end, let $\mathbf{s}^{\prime}$ be the resulting new contract. Then $\mathbf{s}^{\prime} \leq \mathbf{s}$. Also, by construction:

$$
k_{l}(\mathbf{s}, \mathcal{H})=k_{l}\left(\mathbf{s}^{\prime}, \mathcal{H}+i j\right), \forall l \in \mathcal{N} .
$$

So $w(\mathbf{s}, \mathcal{H})=w\left(\mathbf{s}^{\prime}, \mathcal{H}+i j\right)$. That establishes the claim.

\section{B.10 Proof of Proposition 5.5}

Proof Let $t_{1}, t_{2}, \cdots, t_{n_{k}}$ be the shares of workers on the same level, say $N^{k}$, in an optimal contract $\mathbf{s}$. Let $y=\sum_{l>k} \sum_{j \in N^{l}} k_{j}$. Then by Theorem 4.3 , the responsive 
coefficients of these $n_{k}$ members are given by $g\left(t_{i}, y\right)$, and the contribution to welfare by these workers is

$$
G\left(t_{1}, \cdots, t_{n_{k}}\right):=\sum_{i=1}^{n_{k}}\left\{g\left(t_{i}, y\right)-\frac{1}{2} g\left(t_{i}, y\right)^{2}\right\}
$$

Define $F\left(t_{1}, \cdots, t_{n_{k}}\right):=\sum_{i=1}^{n_{k}}\left\{g\left(t_{i}, y\right)\right\}$ as the sum of the responsive coefficients. We prove the proposition by contradiction.

If $t_{m} \neq t_{n}$ for $m \neq n$, then let $s=\frac{\sum_{i=1}^{n_{k}} t_{i}}{n_{k}}$ be the new equalized share for members in $N^{k}$. We claim that

$$
G\left(t_{1}, \cdots, t_{n_{k}}\right)<G(s, \cdots, s) \text { and } F\left(t_{1}, \cdots, t_{n_{k}}\right)<F(s, \cdots, s)
$$

Note $g(t, y)$ is strictly concave in $t$ (Lemma A.1), so

$F\left(t_{1}, \cdots, t_{n_{k}}\right)=n_{k} \sum_{i=1}^{n_{k}}\left\{\frac{1}{n_{k}} g\left(t_{i}, y\right)\right\}<n_{k}\left\{g\left(\frac{\sum_{i=1}^{n_{k}} t_{i}}{n_{k}}, y\right)\right\}=n_{k} g(s, y)=F(s, \cdots, s)$

by Jensen's inequality.

Similarly, $g(t, y)-\frac{1}{2} g(t, y)^{2}=\left(1-\frac{1}{2} t\right) g(t, y)-\frac{1}{2} t y$ is concave in $t$ because

$$
\left(g(t, y)-\frac{1}{2} g(t, y)^{2}\right)^{\prime \prime}=\left(1-\frac{1}{2} t\right) g_{x x}(t, y)-g_{x}(t, y)<0
$$

Here $t<1,1-\frac{1}{2} t>0, g_{x x}<0, g_{x}>0$. By the same logic, we can show $G\left(t_{1}, \cdots, t_{n_{k}}\right)<G(s, \cdots, s)$.

For Equation 23), we can get a similar contradiction as shown in the proof of Theorem 4.14. We do not repeat the argument here.

\section{B.11 Proof of Proposition 5.6}

Proof Let $\mathbf{s}$ be the optimal share profile for $\phi(t, \mathcal{H})$. We compare the coefficients $k_{i}$ for $\mathbf{s}$ under $\mathcal{H}$ and $\mathcal{H}^{\prime}$. The incentives of workers in $N^{k+1}, \cdots, N^{h}$ and $N^{k} \backslash\{i\}$ are obviously the same in both cases. Worker $i$ 's incentive is different because $i$ has more followers, so

$$
k_{i}^{\prime}\left(\mathbf{s}, \mathcal{H}^{\prime}\right)=g\left(s_{i},\left(\sum_{j \in N^{k} \backslash\{i\},} k_{l}\left(\mathbf{s}, \mathcal{H}^{\prime}\right)\right)+y\right)>k_{i}\left(\mathbf{s}, \mathcal{H}^{\prime}\right)=g\left(s_{i}, y\right)
$$


where $y=\sum_{l=k+1}^{h} \sum_{j \in N^{l}} k_{j}(\mathbf{s}, \mathcal{H})$. Therefore, we can reduce the share of $s_{i}$ by a small amount $\delta>0$, such that

$$
g\left(s_{i}-\delta,\left(\sum_{j \in N^{k} \backslash\{i\},} k_{l}\left(\mathbf{s}, \mathcal{H}^{\prime}\right)\right)+y\right)=k_{i}\left(\mathbf{s}, \mathcal{H}^{\prime}\right)=g\left(s_{i}, y\right)
$$

With this reduction, the incentives for workers in $N^{k}$ are also the same as before. Then by induction, the responsive coefficients for workers in $N^{k-1}, \cdots, N^{1}$ are also the same. Therefore, we can find a share profile for $\mathcal{H}^{\prime}$ that uses less total share (adds up to $1-\delta$ ) and yields the same welfare. Extra share is welfare improving by Theorem 4.9 , hence $\phi(t, \mathcal{H})<\phi\left(t, \mathcal{H}^{\prime}\right)$.

\section{B.12 Proof of Equation 11 and Proposition 6.2}

Proof: Once we have equation 11, Proposition 6.2 follows from a similar argument. So, it suffices to show equation 11 holds for any hierarchy in $\mathcal{M}^{s}(N, K, 1)$.

Suppose $\mathcal{H} \in \mathcal{M}^{s}(N, K, 1)$. Take any middle manger, say $M$ with his $q$ followers. Suppose shares of the middle manager and his followers are $u, v_{1}, v_{2}, \cdots, v_{q}$. Then we claim that:

$$
g\left(u, \sum_{i=1}^{q} v_{i}\right)+\sum_{i=1}^{q} v_{i} \leq \frac{4}{3}\left(u+\sum_{i=1}^{q} v_{i}\right)
$$

Taking summation over all the middle level workers, then the right hand side will be $\frac{4}{3}$ times the sum of shares of $2 p$ followers, which is exactly $\frac{4}{3}(1-l)$. The left hand side will be the sum of the responsive coefficients of those $2 p$ workers. That is exactly equation 11 . Inequality $(\dagger)$ follows from

$$
g(x, y)+y \leq \max _{t \in[0, x+y]} g(x+y-t, t)+t=g(x+y-t, t)+\left.t\right|_{t=\frac{2}{3}(x+y)}=\frac{4}{3}(x+y),
$$

while the equality in the middle follows from part 4 of Lemma A.4.

If any middle level worker has more than one follower, then either equation 11 is strict, or equation 12 is strict. In this case we have a strict welfare comparison, which shows that $\mathcal{H}^{1}$ is the most efficient hierarchy in $\mathcal{M}^{s}(N, K, 1)$.

Remark 8 The proof also shows that to achieve the highest welfare under $\mathcal{H}^{1}$, we must assign $m=\frac{1-l}{3 p}$ to each manager and $f=\frac{2(1-l)}{3 p}$ to each terminal worker, and 
$l$ to the leader for some $l \in(0,1)$. The only unknown variable is the number $l$. The optimal l can be determined by solving the corresponding welfare maximization program.

\section{Additional Materials}

\section{C.1 Adding Links is Not Always Welfare Improving}

Take a hierarchy as shown in figure 10a, in which $\mathrm{A}$ and $B_{9}$ are on level 1 , and $B_{j}$ are on level $10-j$ for each $j$. Their shares are: $s_{A}=91 \%$ and $s_{B_{i}}=1 \%, i=$ $1, \cdots, 9$. The responsive coefficients $k_{B_{i}}$ are: $\{0.01,0.016,0.022,0.027,0.033,0.038$, $0.043,0.049,0.054\}$, and $k_{A}=s_{A}=0.9$. If one additional link is added from

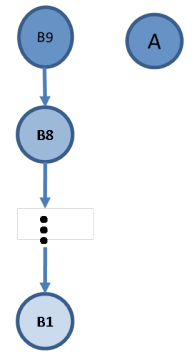

(a) old hierarchy

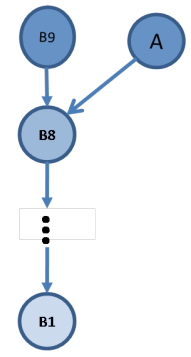

(b) new hierarchy

Figure 10: A counterexample: adding links is not always welfare improving.

A to $B_{8}$ as shown in Figure $10 \mathrm{~b}$, then A's responsive coefficient under the new structure is $\tilde{k}_{A}=g\left(0.9, \sum_{i=1}^{8} k_{B_{i}}\right)=1.10688$, which is further away from $k^{F B}=1$ than $k_{A}=0.9$. Moreover, this transformation only affects the incentive for $\mathrm{A}$; the equilibrium efforts for other members are not affected. Therefore the aggregate welfare of the new hierarchy with this additional link is actually lower than the original one. This is not inconsistent with Theorem 5.3 because we have not adjusted the shares optimally here. 


\section{C.2 More About Belief Functions in V Structure}

We explore more possible belief functions in the $\mathrm{V}$ structure in this subsection. Unlike the analysis with the chain structure, conditions $\left(P 1^{\prime}\right),\left(P 2^{\prime}\right),\left(B^{\prime}\right)$ are not enough to uniquely pin down the equilibrium efforts in this case. In general, different belief functions can support different equilibrium efforts, and one belief function can support multiple equilibria.

Definition 8 Given a belief function $\beta$ satisfying $B^{\prime}$, we say $e_{1}, e_{2}$ are supported by $\beta$ if $e_{1}, e_{2}$ and $\beta$ satisfy conditions $S, P 1^{\prime}, P 2^{\prime}$.

To find equilibria, it suffices to find functions $e_{1}, e_{2}$ that can be supported by a given $\beta$. In the following examples, we list some special belief functions and find the effort functions that can be supported by each.

The first one is the one used in the paper.

1. Pessimistic belief

$$
\beta^{p}\left(\theta_{1}, \theta_{2}\right)=\min \left(\theta_{1}, \theta_{2}\right)
$$

In this case, using FOC, we can rewrite $P 1^{\prime}, P 2^{\prime}$ as:

$$
\forall \theta, \frac{\theta}{3} \leq e_{i}(\theta) \leq \frac{\theta}{3}\left(1+\frac{1}{3 e_{i}^{\prime}(\theta)}\right), \quad i=1,2 .
$$

There are multiple solutions to equation 25. For example, $e_{i}(\theta)=k_{i} \theta$, for any $k_{i} \in\left[\frac{1}{3}, \frac{1+\sqrt{5}}{6}\right], i=1,2$. Nevertheless, the solutions to equation 25 are bounded in the following sense.

Lemma C.1 If $e_{i}$ is monotonic and satisfies equation 25, then

$$
\forall \theta, \underline{e}(\theta) \leq e_{i}(\theta) \leq \bar{e}(\theta)
$$

Here, $\bar{e}(\theta):=g\left(\frac{1}{3}, \frac{1}{3}\right) \theta$ and $\underline{e}(\theta):=\frac{\theta}{3}$ are defined in section 2. So, $\bar{e}(\theta)$ and $\underline{e}(\theta)$ are the upper and lower bounds for the solutions to equation 25. Moreover, both $\bar{e}(\theta)$ and $\underline{e}(\theta)$ satisfy equation 25 .

2. Trigger belief

$$
\beta^{t}\left(\theta_{1}, \theta_{2}\right)= \begin{cases}\theta_{1} & \text { if } \theta_{1}=\theta_{2} \\ 0 & \text { otherwise }\end{cases}
$$


In this case both leaders have the same information about the state. If any leader deviates, $\mathrm{F}$ will detect it and choose zero effort under this belief assumption. Moreover, $P 1^{\prime}, P 2^{\prime}$ are equivalent to:

$$
\left(e_{i}(\theta)-\frac{1}{3} \theta\right)^{2} \leq \frac{2}{9} \theta^{2}, \quad i=1,2 .
$$

In particular, $e_{i}(\theta)=k_{i} \theta$, for any $k_{i} \in\left(0, \frac{1+\sqrt{2}}{3}\right], i=1,2$ will satisfy these conditions. Not all solutions to the above equations are linear.

3. Weighted belief

$$
\beta^{w 1, w 2}\left(\theta_{1}, \theta_{2}\right)=w_{1} \theta_{1}+w_{2} \theta_{2}
$$

for $0 \leq w_{i} \leq 1, w_{1}+w_{2}=1$. Under this belief, $P 1^{\prime}, P 2^{\prime}$ can be written as:

$$
\frac{\theta}{3}\left(1+\frac{w_{i}}{3 e_{i}^{\prime}(\theta)}\right)=e_{i}(\theta), \quad i=1,2 .
$$

The solutions to this differential equation are linear in $\theta$, and given by

$$
e_{i}\left(\theta \mid w_{i}\right)=g\left(\frac{1}{3}, \frac{w_{i}}{3}\right) \theta, i=1,2 .
$$

Note that $\underline{e}$ and $\bar{e}$ are special cases with $w_{i}=0$, and $w_{i}=1$. But, this belief function cannot support $e_{i}=\underline{e}, \forall i$ or $e_{i}=\bar{e}, \forall i$, because otherwise $w_{1}+w_{2} \neq 1$.

Not every specification of $\beta$ is consistent with equilibrium, as the next example shows.

4. Optimistic belief

$$
\beta^{o}\left(\theta_{1}, \theta_{2}\right)=\max \left(\theta_{1}, \theta_{2}\right)
$$

In this case, $P 1^{\prime}, P 2^{\prime}$ can be written as:

$$
\frac{\theta}{3} \geq e_{i}(\theta) \geq \frac{\theta}{3}\left(1+\frac{1}{3 e_{i}^{\prime}(\theta)}\right), \quad i=1,2 .
$$

Note that $\frac{\theta}{3}<\frac{\theta}{3}\left(1+\frac{1}{3 e_{i}^{\prime}(\theta)}\right)$. These two inequalities are inconsistent, hence there is no separating equilibrium with optimistic belief. 
The trigger belief $\beta^{t}$ can generate not only very efficient outcomes, for example $e_{i}(\theta)=\frac{1+\sqrt{2}}{3} \theta, i=1,2$, but also very inefficient ones, for example $e_{i}(\theta)=\epsilon \theta, i=$ 1,2 for arbitrarily small $\epsilon>0$. A unsatisfactory fact about $\beta^{t}$ is that it has jumps and it is not monotonic in $\theta_{1}, \theta_{2}$. For $\beta^{p}$, and $\beta^{w 1, w 2}$, the equilibrium efforts of both leaders are at least $\frac{\theta}{3}$.

Sometimes, the following restrictions are natural to assume.

$(B D M) \quad \beta$ is continuous, differentiable and monotonic in $\theta_{1}, \theta_{2}$

With this assumption (BDM), $P 1^{\prime}$ and $P 2^{\prime}$ can be replaced by the corresponding FOCs.

$$
\frac{\theta}{3}\left(1+\frac{\beta_{i}(\theta, \theta)}{3 e_{i}^{\prime}(\theta)}\right)=e_{i}(\theta), i=1,2
$$

where $\beta_{i}=\frac{\partial \beta}{\partial \theta_{i}}$. For any $\beta$ satisfying (BDM), we can find an equilibrium by solving the differential equations (31). Although the solutions are in general different for different $\beta$ s, nevertheless, we claim:

Claim C.2 All solutions to equation 31 satisfy equation 25.

Proof Differentiating both sides of $\beta(\theta, \theta)=\theta$ gives $\beta_{1}(\theta, \theta)+\beta_{2}(\theta, \theta)=1$. Note $\beta$ is monotonic, so $\beta_{i}(\theta, \theta) \geq 0$. Therefore $0 \leq \beta_{i}(\theta, \theta) \leq 1$. By equation 31.

$$
\frac{\theta}{3}=\frac{\theta}{3}\left(1+\frac{0}{3 e_{i}^{\prime}(\theta)}\right) \leq e_{i}(\theta)=\frac{\theta}{3}\left(1+\frac{\beta_{i}(\theta, \theta)}{3 e_{i}^{\prime}(\theta)}\right) \leq \frac{\theta}{3}\left(1+\frac{1}{3 e_{i}^{\prime}(\theta)}\right)
$$

which is exactly equation 25 .

This immediately yields the following result.

Proposition C.3 If $\left\{e_{i}, i=1,2\right\}$ can be supported by a belief function $\beta$ satisfying $(B D M)$, then $\left\{e_{i}, i=1,2\right\}$ can also supported by pessimistic belief $\beta^{p}$.

Of course, $\beta$ is part of the equilibrium. In general, we cannot impose assumptions on the endogenous belief functions. We believe that (BDM) is satisfied by a large class of belief functions, although $\beta^{t}, \beta^{p}$ violate (BDM). Moreover, we can show that Proposition C.3 also holds for $\beta$ satisfying a weaker differentiability condition than (BDM): existence of left and right derivatives (not necessarily equal). 
In particular, pessimistic belief $\beta^{p}$ satisfies this weaker condition. In the text we argued that assuming $\beta^{p}$ as the out-of-equilibrium belief of $\mathrm{F}$ makes some sense. In section 2, we found the upper and lower bounds on the corresponding welfare with $\beta^{p}$. By Proposition C.3, these upper and lower bounds hold for any belief satisfying $(\mathrm{BDM})$ or a weaker differentiability condition. ${ }^{22}$

\section{Proof of Lemma C.1}

Proof It is easy to see that $e_{i}(\theta) \geq \frac{\theta}{3}$. If $e_{i}(\theta) \geq g\left(\frac{1}{3}, \frac{1}{3}\right) \theta$, then equation 25 implies $g\left(\frac{1}{3}, \frac{1}{3}\right) \leq \frac{e_{i}(\theta)}{\theta} \leq \frac{1}{3}\left(1+\frac{1}{3 e_{i}^{\prime}(\theta)}\right)$. Equivalently, $e_{i}^{\prime}(\theta) \leq g\left(\frac{1}{3}, \frac{1}{3}\right)$. Let $f(\theta)=$ $e_{i}(\theta)-g\left(\frac{1}{3}, \frac{1}{3}\right) \theta$. So $f^{\prime}(\theta) \leq 0$ whenever $f(\theta) \geq 0$. Also $e_{i}(0)=0$, so $f(0)=0$. Lemma A.5 shows that $f(\theta) \leq 0, \forall \theta$, or equivalently $e_{i}(\theta) \leq g\left(\frac{1}{3}, \frac{1}{3}\right) \theta, \forall \theta$.

\section{References}

[1] D. Acemoglu and M.O. Jackson, History, expectations, and leadership in the evolution of social norms, Tech. report, National Bureau of Economic Research, 2011.

[2] James Andreoni, Leadership giving in charitable fund-raising, Journal of Public Economic Theory 8 (2006), no. 1, 1-22.

[3] Venkatesh Bala and Sanjeev Goyal, Learning from neighbours, The Review of Economic Studies 65 (1998), no. 3, 595-621.

[4] A.V. Banerjee, A simple model of herd behavior, The Quarterly Journal of Economics 107 (1992), no. 3, 797.

[5] S. Bikhchandani, D. Hirshleifer, and I. Welch, A theory of fads, fashion, custom, and cultural change as informational cascades, Journal of political Economy (1992), 992-1026.

[6] P. Bolton, M.K. Brunnermeier, and L. Veldkamp, Leadership, coordination and mission-driven management, 2008.

\footnotetext{
${ }^{22}$ However, for trigger belief $\beta^{t}$, we can support $e_{i}=\frac{1+\sqrt{2}}{3} \theta, i=1,2$. The corresponding welfare is $\frac{(11+8 \sqrt{2})}{18} \theta^{2} \approx 1.23965 \theta^{2}$, which is higher than $W^{S}$.
} 
[7] _ Economists' perspectives on leadership, Handbook of Leadership Theory and Practice, Harvard Business School Press, 2010, p. 239.

[8] P. Bolton and M. Dewatripont, The firm as a communication network, The Quarterly Journal of Economics 109 (1994), no. 4, 809.

[9] G.A. Calvo and S. Wellisz, Supervision, loss of control, and the optimum size of the firm, The Journal of Political Economy (1978), 943-952.

[10] _ Hierarchy, ability, and income distribution, The Journal of Political Economy (1979), 991-1010.

[11] I.K. Cho and D.M. Kreps, Signaling games and stable equilibria, The Quarterly Journal of Economics 102 (1987), no. 2, 179.

[12] L. Garicano, Hierarchies and the organization of knowledge in production, Journal of Political Economy 108 (2000), no. 5, 874-904.

[13] B.E. Hermalin, Toward an economic theory of leadership: Leading by example, The American Economic Review 88 (1998), no. 5, 1188-1206.

[14] _ Leadership and corporate culture, forthcoming Handbook of Organization (2007).

[15] _ Leading for the long term, Journal of Economic Behavior \& Organization 62 (2007), no. 1, 1-19.

[16] B. Holmstrom, Moral hazard in teams, The Bell Journal of Economics 13 (1982), no. 2, 324-340.

[17] M.O. Jackson, Social and economic networks, Princeton Univ Press, 2008.

[18] M. Komai and M. Stegeman, Leadership based on asymmetric information, The RAND Journal of Economics 41 (2010), no. 1, 35-63.

[19] M. Komai, M. Stegeman, and B.E. Hermalin, Leadership and information, The American Economic Review 97 (2007), no. 3, 944-947. 
[20] G.J. Mailath, Incentive compatibility in signaling games with a continuum of types, Econometrica (1987), 1349-1365.

[21] T. Marschak and S. Reichelstein, Communication requirements for individual agents in networks and hierarchies, 1995.

[22] _ Network mechanisms, informational efficiency, and hierarchies, Journal of Economic Theory 79 (1998), no. 1, 106-141.

[23] P. Milgrom and C. Shannon, Monotone comparative statics, Econometrica (1994), 157-180.

[24] J. Potters, M. Sefton, and L. Vesterlund, Why announce leadership contributions?: An experimental study of the signaling and reciprocity hypotheses, Tilburg University, 2001.

[25] - After you-endogenous sequencing in voluntary contribution games, Journal of Public Economics 89 (2005), no. 8, 1399-1419.

[26] A. Prat, Hierarchies of processors with endogenous capacity, Journal of Economic Theory 77 (1997), no. 1, 214-222.

[27] Y. Qian, Incentives and loss of control in an optimal hierarchy, The Review of Economic Studies 61 (1994), no. 3, 527.

[28] M. Rothschild and J.E. Stiglitz, Increasing risk: I. a definition, Journal of Economic theory 2 (1970), no. 3, 225-243.

[29] T. Van Zandt, Real-time decentralized information processing as a model of organizations with boundedly rational agents, The Review of Economic Studies 66 (1999), no. 3, 633.

[30] L. Vesterlund, The informational value of sequential fundraising, Journal of Public Economics 87 (2003), no. 3-4, 627-657.

[31] J.B.I. Vidal and M. Möller, When should leaders share information with their subordinates?, Journal of Economics \& Management Strategy 16 (2007), no. 2, 251-283. 
[32] E. Winter, Incentives and discrimination, The American Economic Review 94 (2004), no. 3, 764-773.

[33] _ Optimal incentives for sequential production processes, The RAND Journal of Economics 37 (2006), no. 2, 376-390.

[34] - Transparency and incentives among peers, The RAND Journal of Economics 41 (2010), no. 3, 504-523. 